\title{
Adaptive Multiuser Detection and Beamforming for Interference Suppression in CDMA Mobile Radio Systems
}

\author{
Samir Kapoor, Member, IEEE, Sridhar Gollamudi, Member, IEEE, Shirish Nagaraj, Student Member, IEEE, \\ and Yih-Fang Huang, Fellow, IEEE
}

\begin{abstract}
This paper considers the problem of interference suppression in direct-sequence code-division multiple-access (DSCDMA) systems over fading channels. An adaptive array receiver is presented which integrates multiuser detection, beamforming, and RAKE reception to mitigate cochannel interference and fading. The adaptive multiuser detector is formulated using a blind constrained energy minimization criterion and adaptation is carried out using a novel algorithm based on set-membership parameter estimation theory. The proposed detector overcomes the shortcomings of conventional LMS- and RLS-type algorithms, namely, that of slow convergence and large computational load, respectively. This is especially the case when strong interferers are present or when the number of adaptive weights is relatively large. DS-CDMA systems can have a relatively large number of spatially distributed interferers. Thus beamforming is based on direction-of-arrival (DOA) estimates provided by an approximate maximum-likelihood estimator (DOA-MLE). Unlike previous approaches, the DOA-MLE exploits the structure of the DS-CDMA signaling scheme resulting in robust performance and simple implementation in the presence of angle spreading. The overall method is suitable for real-time implementation and can substantially improve the interference suppression capabilities of a CDMA system.
\end{abstract}

Index Terms - Adaptive filters, array signal processing, code division multiaccess, direction of arrival estimation, interference suppression, mobile communication.

\section{INTRODUCTION}

$\mathbf{T}$ THE USE OF spread-spectrum multiple-access techniques is well established in the wireless communications arena. In particular, direct-sequence code-division multiple access (DS-CDMA) has been widely studied in the literature and has been implemented in several commercial systems as well. Adaptive interference suppression techniques based on multiuser detection (MUD) and antenna array processing have

Manuscript received June 22, 1997; revised April 20, 1998. This work was supported in part by the National Science Foundation under Grant MIP9705173, in part by the Center for Applied Mathematics at the University of Notre Dame, and in part by Tellabs Research Center, Mishawaka, IN. Parts of this paper were presented at the First IEEE Workshop on Signal Processing Advances in Wireless Communications (SPAWC'97), Paris, France, April 1997, and at the 34th Allerton Conference on Communication, Control and Computing, Monticello, IL, October 1996.

S. Kapoor was with the Department of Electrical Engineering, University of Notre Dame, Notre Dame, IN 46556 USA. He is now with NEC USA, Inc., Princeton, NJ USA.

S. Gollamudi, S. Nagaraj, and Y. F. Huang are with the Department of Electrical Engineering, University of Notre Dame, Notre Dame, IN 46556 USA.

Publisher Item Identifier S 0018-9545(99)07373-9. recently been considered as powerful methods for increasing the quality, capacity, and coverage of these systems. They provide a superior, though computationally more expensive, alternative to conventional single-sensor matched filtered detection which is severely limited by multiaccess interference (MAI).

This paper focuses on cochannel interference (CCI) mitigation techniques for the uplink channel of DS-CDMA systems over slowly fading channels. CCI is known to be the dominant impairment for such systems which ultimately limits the achievable performance and is exacerbated by the large fluctuations in received signal power due to fading and distance (the near-far effect). Multiuser detection [13], [18], [29] and adaptive array processing [3], [21], [22], [27], [32] have been shown to be promising solutions to this problem. Toward this end, a new adaptive array receiver structure is presented which adopts a two-pronged approach to CCI suppression using MUD and beamforming. Also, a RAKE structure is incorporated for operation over frequency-selective fading channels. Thus the overall receiver makes combined use of frequency diversity through the use of RAKE combining, code (or time) diversity and angle diversity through the use of beamforming.

\section{A. Multiuser Detection for CCI Suppression}

The near-far problem is a major hurdle for DS-CDMA systems and power control has been the method of choice for current systems such as IS-95 [23]. While some basic power control is essential for the operation of any mobile radio system, precise power control methods for overcoming the near-far problem have several drawbacks. They are wasteful of bandwidth and power, increase the complexity of the mobile transceiver, and most importantly, do not provide very reliable and satisfactory performance. When MUD schemes are used, a centralized receiver can receive CDMA signals from different users with large power differences, and yet detect their signals with error probabilities that approach that of optimal detection in the absence of MAI. The basis for most such methods is to exploit the known structure of the signal and interference, i.e., the signal from each user consists of products of signature waveforms with information symbols from a known alphabet.

The principles of MUD are by now well established [13], [29]. The optimal detector [29] is a major theoretical milestone but has limited practical utility due to its exponential 
complexity in the number of users. Decorrelating detectors have enjoyed much popularity due to their near-far resistant performance and much reduced linear complexity [29]. A minimum energy formulation for blind adaptive near-far resistant MUD was introduced in [13] and also used in [27]. While the minimum mean square error (MMSE) and the equivalent minimum output energy solutions are known to be near-far resistant, a major obstacle lies in adaptively seeking the optimum solutions. Stochastic gradient algorithms such as LMS are very attractive because of their simplicity but suffer from poor convergence due to the large dimensionality in typical CDMA systems and the fluctuation in received signal powers [18]. Least squares (LS) algorithms, on the other hand, provide adequate convergence speed under these circumstances, but can be prohibitively complex to implement. Note that most low-complexity fast-RLS-type algorithms are not applicable due to the lack of time-shift structure in the input vectors [12]. Thus there is a clear imperative to develop reduced-complexity MUD schemes without having to sacrifice performance. The major difference in the adaptive MUD detector presented in this paper is that it utilizes a novel recursive algorithm based on set-membership parameter estimation theory [4], [8] which provides significant benefits in terms of tracking, convergence, and complexity.

\section{B. Multichannel CDMA Reception}

The use of antenna arrays offers the possibility of utilizing the spatial characteristics of different user signals to augment the temporal discrimination provided by their signature sequences. Adaptive arrays are typically designed for either utilizing spatial diversity for mitigating the effect of fading or for using the inherent angle diversity in the received signals for CCI reduction. In [17], an adaptive array receiver configured as the cascade of a beamformer and matched filter (MF) detector is presented. The update of the beamformer weights in this structure is rather cumbersome, especially over fading channels. A receiver structure with an MF detector followed by a beamformer is presented in [21]. However, the eigen-decomposition-based DOA estimation algorithm used for beamforming is computationally complex. An extension of this receiver structure, called a $2 \mathrm{D}-\mathrm{RAKE}$ is also presented [21], [22] while noncoherent RAKE combining with $M$-ary orthogonal modulation is considered in [22]. Joint spatiotemporal reception, akin to broadband beamforming [28], has also been considered [3], [18]. A drawback of this approach is the large number of adaptive weights, which is given by the product of the number of chips per symbol (assuming chip rate sampling) and the number of antenna elements. Such configurations can also be sensitive to imperfect knowledge of the array manifold, particularly when angle spreading is present [3].

In this paper, a multisensor receiver is used with a MUD for each element in the first stage. This is followed by a bank of DOA-based beamformers and RAKE combining for frequency selective channels (Fig. 1). For flat fading channels, the receiver structure simplifies to a single beamformer and single tap equalizer. Since RAKE combining takes place after beamforming for each branch, channel fading estimation can take place after MAI has been suppressed. Also, the beamformer operates on symbol-rate samples after MUD. This facilitates the development of a new approximate maximum-likelihood DOA estimation (DOA-MLE) algorithm.

This paper is organized as follows. Section II presents the CDMA signal model and the problem formulation. The MUD technique is developed in Section III. This is followed by a description of the DOA estimation method, beamforming and RAKE combining in Section IV and its performance analysis. Numerical results are presented in Section V along with some discussions and the paper is concluded in Section VI.

\section{CDMA Signal Model AND PROBlem Formulation}

Consider an asynchronous DS-CDMA system with $K$ users. Asynchronous uplink transmissions are received at a centralized receiver from all cochannel active users within the cell of interest and from neighboring cells as well. Let the information symbol sequence from the $k$ th user be denoted by $A_{k}(\cdot)$, chosen in general from a complex alphabet. Assuming the symbol and chip duration to be $T_{s}$ and $T_{c}$, respectively, resulting in a nominal processing gain $N=T_{s} / T_{c}$, the spreadspectrum signal emanating from the $k$ th user is given by

$$
v_{k}(t)=\sum_{n} \sqrt{P_{k}} A_{k}\left(\left\lfloor\frac{n}{N}\right\rfloor\right) c_{k}(n \% N) \delta\left(t-n T_{c}\right)
$$

where $n$ is the chip index; \% denotes the modulus operation and \lfloor\rfloor denotes the floor operation; $P_{k}$ is the transmitted power of the $k$ th user; $c_{k}(n \% N)$ denotes the $n$th chip of the $k$ th users' periodic spread-spectrum sequence with period $N$. After performing baseband pulse shaping with a filter $\psi(t)$, the transmitted waveform is given by

$$
x_{k}(t)=\sum_{n} \sqrt{P_{k}} A_{k}\left(\left\lfloor\frac{n}{N}\right\rfloor\right) c_{k}(n \% N) \psi\left(t-n T_{c}\right)
$$

The pulse $\psi(t)$ is assumed to have unit energy and duration $T_{c}$. Assume that the transmitted signal from the $k$ th user is received at an antenna array receiver with $M$ elements. An $L$-path frequency-selective slowly fading model is assumed in order to formulate the spatiotemporal impulse response of the $m$ th sensor to the signals from the $k$ th user (see Appendix A) according to

$$
h_{k}^{(m)}(t)=\sum_{l=0}^{L-1} a_{k l}^{(m)} \delta\left(t-\tau_{k l} T_{c}\right)
$$

where $a_{k l}^{(m)}$ denotes the composite response of the $m$ th sensor to the $l$ th multipath component from the $k$ th user; $\tau_{k l} T_{c}$ is the time delay of the $l$ th multipath component of the $k$ th user's received signal and that the maximum delay $\tau_{k(L-1)} T_{c}<T_{s}$. Thus the received complex baseband signal from the $k$ th user 


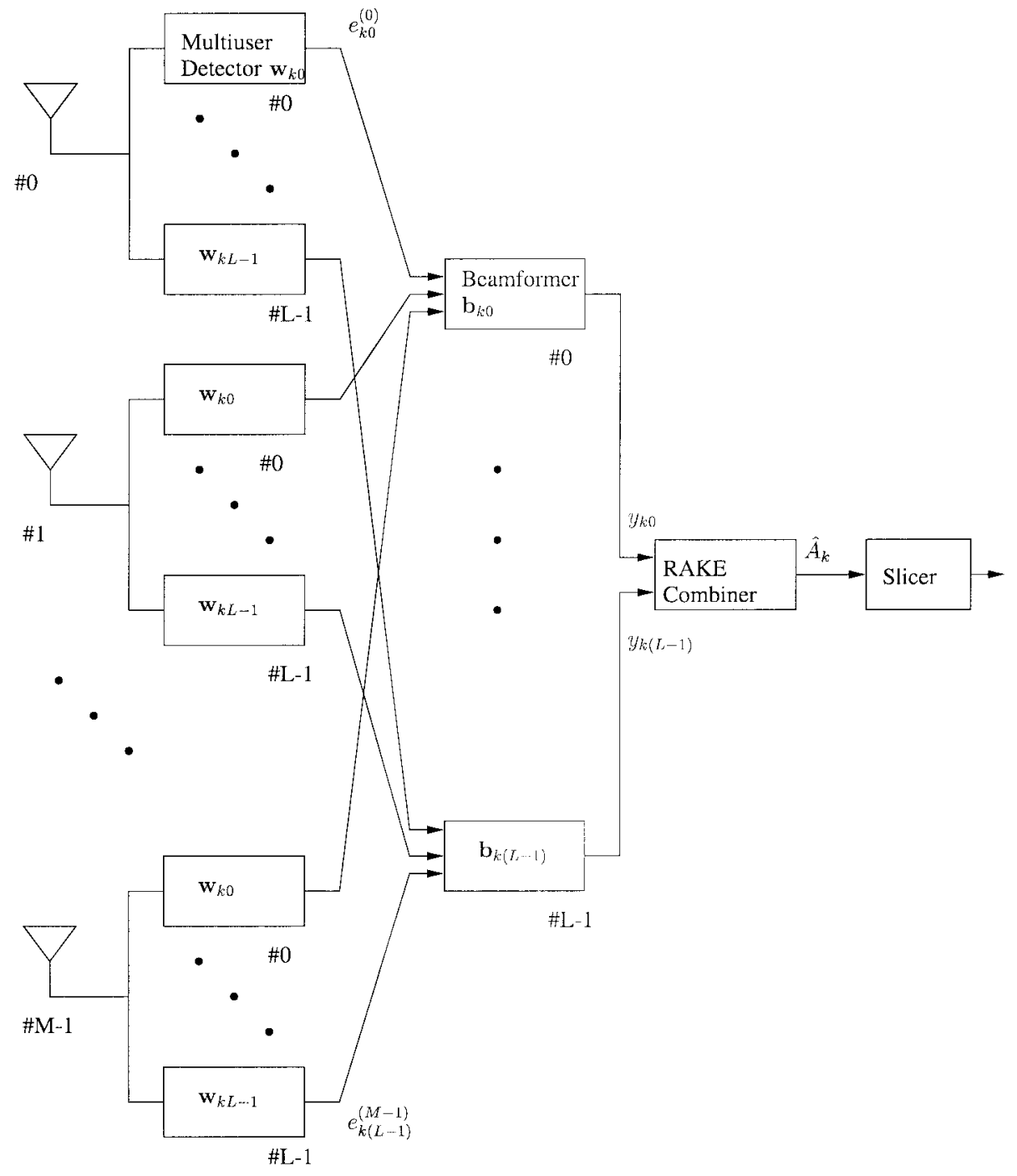

Fig. 1. Receiver structure for $L$-path frequency selective fading channel for $k$ th user.

at the $m$ th element is given by the convolution

$$
\begin{aligned}
r_{k}^{(m)}(t)= & x_{k}(t) * h_{k}^{(m)}(t) \\
= & \sum_{l=0}^{L-1} a_{k l}^{(m)} x_{k}\left(t-\tau_{k l} T_{c}\right) \\
= & \sum_{n} \sqrt{P_{k}} A_{k}\left(\left\lfloor\frac{n}{N}\right\rfloor\right) c_{k}(n \% N) \\
& \cdot \sum_{l=0}^{L-1} a_{k l}^{(m)} \psi\left(t-n T_{c}-\tau_{k l} T_{c}\right) .
\end{aligned}
$$

The received signal from $K$ asynchronous users at the centralized receiver is obtained as the superposition of each user's signals according to

$$
r^{(m)}(t)=\sum_{k=0}^{K-1} r_{k}^{(m)}\left(t-\nu_{k}\right)+n^{(m)}(t)
$$

where $\nu_{k}$ is the flat propagation delay of the $k$ th user and $n^{(m)}(t)$ denotes the $m$ th sensor's front-end additive noise. The receiver structure shown in Fig. 1 is used to recover transmitted symbols from all desired users. For a particular desired user, the composite signal is chip match filtered and fed into multiple RAKE arms, each delayed by a chip time or more. It is assumed that the receiver uses conventional techniques for determining which RAKE arms contain delayed copies of the signal [21]. Also, RAKE receiver arms for user $k$ are synchronized to the path delays $\tau_{k l} T_{c}$. Without loss of generality, chip synchronous reception is assumed and nonidealities due to imperfect carrier synchronization and clock jitter are ignored. Thus the chip rate samples for the $k$ th user with delay $\tau_{k l} T_{c}$ are obtained at the $m$ th sensor after demodulation as

$$
\hat{v}_{k l}^{(m)}(n)=\left.r^{(m)}(t) * \psi\left(-\left(t-\tau_{k l} T_{c}-\nu_{k}\right)\right)\right|_{t=n T_{c}} .
$$

Denoting the sequence of received samples of dimension $N$ spanning one symbol as $\hat{v}_{k l}^{(m)}$, the chip sample vector for the 
$p$ th symbol of the desired user, i.e., for $n \in[p N, p N+N-1]$, is given by

$$
\begin{aligned}
\boldsymbol{v}_{k l}^{(m)}(p)= & \sqrt{P_{k}} a_{k l}^{(m)} A_{k}(p) \boldsymbol{c}_{k} \\
& +\sum_{r \neq l} \sqrt{P_{r}} a_{k r}^{(m)}\left[A_{k, 0} \boldsymbol{c}_{k r, 0}+A_{k,-1} \boldsymbol{c}_{k r,-1}\right] \\
& +\sum_{h \neq k} \sqrt{P_{h}} \sum_{l=0}^{L-1} a_{h l}^{(m)}\left[A_{h, 0} \boldsymbol{c}_{h l, 0}+A_{h,-1} \boldsymbol{c}_{h l,-1}\right] \\
& +\tilde{\boldsymbol{n}}^{(m)}(p)
\end{aligned}
$$

where

$$
\begin{aligned}
c_{k} & =\left[c_{k}(0), c_{k}(1), \cdots, c_{k}(N-1)\right]^{T} \\
\boldsymbol{c}_{k l, 0}= & =\left[0, \cdots, 0, c_{k}(0), \cdots, c_{k}\left(N-\tau_{k l} T_{c}-1\right)\right]^{T} \\
\boldsymbol{c}_{k l,-1} & =\left[c_{k}\left(N-\tau_{k l} T_{c}\right), \cdots, c_{k}(N-1), 0, \cdots, 0\right]^{T}
\end{aligned}
$$

and $A_{k, 0}$ and $A_{k,-1}$ denote the two overlapping symbols; $\tilde{\boldsymbol{n}}^{(m)}(p)$ is the vector of filtered noise samples during the $p$ th symbol. Thus in addition to the filtered background noise, each sample of the received chip vector for the $p$ th symbol has two interfering components. These arise from the multipath components of the same user with different time delays and from all other users. In addition, each interferer (self-multipath as well as from other users) contributes two independent interference vectors to the received sample vector in each symbol time. The $k$ th users' symbols for the lth RAKE arm are extracted using a linear detector $w_{k l}$, characterized by the discrete-time inner product with the sampled chip sequence as

$$
e_{k l}^{(m)}(p)=\boldsymbol{w}_{k l}^{H} \hat{\boldsymbol{v}}_{k l}^{(m)}(p) .
$$

As can be seen in Fig. 1, a linear MUD is used for each RAKE branch at all array elements. The computation and adaptive update of these linear detectors is now addressed.

\section{Blind Multiuser DeteCtion}

The goal here is to blindly compute linear decorrelation weight vectors to preserve the desired signal and mitigate interference, see, e.g., [9], [13], [27], and [30]. In [13], it is shown that a blind MUD can be formulated using no more information than the conventional detector, i.e., knowing the timing and signature sequence of the desired user. The linear blind MUD is decomposed into two orthogonal components. The first component is "anchored" to the signature sequence of the desired user while the second component is always orthogonal to it. Using the minimum output energy criterion, the latter component can be used to adaptively suppress MAI and blindly achieve the MMSE solution. A conceptually similar approach is described in [27] using constrained beamforming techniques originating in classical array signal processing and is also used in this paper.

For the special case that the receiver treats MAI as additive white noise or when detector adaptivity is not feasible, the linear detector is merely the signature sequence of the desired user. The following algorithm is presented following the framework of the well-known Generalized Sidelobe Canceler
(GSC) [28]. In the rest of this paper, $\boldsymbol{w}_{k}$ denotes the detector vector for any one of the $L$ RAKE arms and the corresponding subscript is dropped for notational brevity. Constraining the detector to present a unit response to the desired user's signature sequence, and decomposing $w_{k}$ into corresponding constrained and unconstrained components

$$
\boldsymbol{w}_{k}=\boldsymbol{w}_{k, q}-\boldsymbol{C}_{k, n} \boldsymbol{w}_{k, a}
$$

where the nonadaptive part of $\boldsymbol{w}_{k}$

$$
\boldsymbol{w}_{k, q}=C_{k}\left(\boldsymbol{C}_{k}^{H} \boldsymbol{C}_{k}\right)^{-1} g
$$

with the constraint matrix $\boldsymbol{C}_{k}=\boldsymbol{c}_{k}$ and output constraint $g=1$. Thus in this case $\boldsymbol{w}_{k, q}=\boldsymbol{c}_{k} / N$. The columns of the $N \times(N-1)$ matrix $\boldsymbol{C}_{k, n}$ span the null space of $\boldsymbol{c}_{k}$. In general, of course, multiple constraints can be imposed on $\boldsymbol{w}_{k, q}$, for instance, to exploit the knowledge of signature sequences and timing of other interfering users and enable faster convergence of adaptive solutions. Such constraints appear as columns of $\boldsymbol{C}_{k}$ and result in a corresponding decrease in the dimensionality of $\boldsymbol{w}_{k, a}$. In the extreme case when all the columns of $C_{k}$ are constrained, the detector ceases to be adaptive. $\boldsymbol{C}_{k, n}$ is readily obtained via one of many orthogonalizing procedures [28]. In fact, for the special case above, $C_{k, n}$ can be precomputed off-line for each desired signature sequence. $\boldsymbol{w}_{k, a}$ denotes the $N$-1-dimensional adaptive portion of $\boldsymbol{w}_{k}$. Thus the output of the $m$ th array element for the lth RAKE arm is given by

$$
e_{k l}^{(m)}(p)=\boldsymbol{w}_{k, q}^{H} \hat{\boldsymbol{v}}_{k l}^{(m)}(p)-\boldsymbol{w}_{k, a}^{H} C_{k, n}^{H} \hat{\boldsymbol{v}}_{k l}^{(m)}(p) .
$$

Equation (3.3) can be viewed as a standard adaptive filtering problem with $\boldsymbol{w}_{k, q}^{H} \hat{\boldsymbol{v}}_{k l}^{(m)}(p)$ serving as the desired signal; $\boldsymbol{C}_{k, n}^{H} \hat{\boldsymbol{v}}_{k l}^{(m)}(p)$ serving as the input vector; $\boldsymbol{w}_{k, a}$ denoting the adaptive weight vector; and $e_{k l}^{(m)}(p)$ denoting the estimation error. When a single signal-preserving constraint is used, the vector $\boldsymbol{w}_{k, a}$ which minimizes the mean squared error (MSE) in (3.3) is given by

$$
w_{k, a}=\left(\boldsymbol{C}_{k, n}^{H} \boldsymbol{R}_{i} \boldsymbol{C}_{k, n}\right)^{-1} \boldsymbol{C}_{k, n}^{H}\left(\boldsymbol{R}_{i}+\boldsymbol{R}_{s i}\right) \boldsymbol{w}_{k, q}
$$

where $\boldsymbol{R}_{\boldsymbol{i}}$ denotes the noise-plus-MAI covariance matrix and $\boldsymbol{R}_{s i}$ denotes the cross-covariance matrix of the desired signal and MAI. The minimum mean squared error (MMSE) is given by $\boldsymbol{w}_{k}^{H} \boldsymbol{R}_{i} \boldsymbol{w}_{k}$ where $\boldsymbol{w}_{k}$ is obtained by substituting $\boldsymbol{w}_{k, a}$ from (3.4) in (3.1).

\section{A. Adaptive Solution Strategies}

The conventional solution to the above is to perform an unconstrained optimization involving $\boldsymbol{w}_{k, a}$ which can be obtained via stochastic gradient descent or LS algorithms [12]. As described in Section I, a desirable goal is to seek an alternative solution strategy which has LS like, or better, properties with reduced computational burden. A compelling solution to this problem is to consider a new approach based on set-membership parameter estimation theory, see, e.g., [4], [7], [8]. Set-membership parameter estimation techniques can lead to recursive algorithms with powerful properties and provide a set of feasible estimates rather than a point estimator. For 
excellent tutorial overviews of set-membership theory, see [4] and [7]. Other key papers detailing the structure, features, convergence and tracking properties, and signal processing applications of set-membership algorithms include [5], [6], [8], and the references therein.

Specifically, the focus of this paper is on a subset of setmembership techniques, namely, the class of optimal bounding ellipsoids (OBE) algorithms [5], [8]. There are several features of OBE algorithms which render them attractive for the problem at hand. Experience has shown that OBE algorithms perform better than weighted recursive LS algorithms in tracking time-varying parameters and in low SNR situations [5], [10], [15]. Furthermore, OBE algorithms are computationally efficient due to their discerning update property. They can also provide an explicit indication of any loss in tracking - a feature not possessed by point estimation algorithms such as LMS or LS algorithms. Although LS algorithms can be equipped with such indicator functions rather easily, such a feature is an integral part of parameter estimation using OBE algorithms. As a point of common ground, the geometric centers of the bounding ellipsoids in OBE algorithms (which are usually taken as point estimates at any given time) are known to be weighted recursive LS estimates [7]. Simply stated, the optimization of the weighting (update) factors of data sets according to set-membership principles essentially leads to the discerning update property and superior convergence and tracking properties.

The OBE algorithms may appear to have $\mathcal{O}\left(N^{2}\right)$ complexity from an inspection of the recursive update equations. However, their discerning update (or data-selective) feature can be fruitfully exploited for significant reduction in complexity [6], [10], [11]. Since the primary interest here lies within the class of linear detectors, the computational complexity of updating the adaptive weights vectors is the differentiating factor. In [10], it is shown that under typical cellular traffic statistics, using the selective update criterion described in Section III-B, approximately $K / 7$ update processors can be statistically shared among $K$ independent users being received at a base station. Another way to exploit the discerning update property for computational savings is described in [6] where an approximately $\mathcal{O}(N)$ average complexity implementation is obtained over a block of bits using time-buffered operation of the update processor for a single user. Thus the structure and properties of OBE algorithms can narrow the gap between performance and complexity which is encountered by several conventional adaptive filtering algorithms.

\section{B. OBE Algorithm}

A recursive algorithm is now derived to estimate $\boldsymbol{w}_{k, a}$ based on set-membership principles using OBE. The idea here is to update the estimator such that the estimation error is constrained to lie within a specified performance bound. This approach is conceptually reminiscent of [1] where a linear programming framework is used for robust adaptive beamforming and a beamformer weight vector is adapted to attain a certain performance bound using a recursive linear programming algorithm. The goal is to construct an OBE algorithm which attempts to seek solution vectors $\boldsymbol{w}_{k, a}$ that meet the following specification for all $p$ :

$$
\sum_{m=0}^{M-1}\left|\boldsymbol{w}_{k, q}^{H} \hat{\boldsymbol{v}}_{k l l}^{(m)}(p)-\boldsymbol{w}_{k, a}^{H} \boldsymbol{C}_{k, n}^{H} \hat{\boldsymbol{v}}_{k, l}^{(m)}(p)\right|^{2} \leq \gamma_{k}^{2}
$$

where $\gamma_{k}^{2}$ is a specified constant corresponding to a desired performance level and may also be viewed as a design parameter. Furthermore, to ensure that the solution to the above is nonempty, the input parameters are assumed to come from a so-called design space $\mathcal{D}$ consisting of all input vectors $\hat{v}_{k l l}^{(m)}(p)$ formed by the additive noise values bounded in magnitude by a suitable constant [20]. Weight vectors which achieve (3.5) for all possible input sequences from the design space constitute the so-called feasibility set and any member of this set is a valid detector. Since this is a worst case deterministic error specification, excellent performance is obtained even for those input vectors arising from outside the design space. The objective of the OBE methodology is to seek this feasibility set or any one of its members. This set is given by

$$
\begin{array}{r}
\Theta_{k}\left(N, \gamma_{k}\right) \triangleq \bigcap_{\hat{\boldsymbol{v}}_{k l}^{(m)}(p) \in \mathcal{D}}\left\{\boldsymbol{w} \in \mathcal{C}^{N-1}: \sum_{m=0}^{M-1} \mid \boldsymbol{w}_{k, q}^{H} \hat{\boldsymbol{v}}_{k l}^{(m)}(p)\right. \\
\left.-\left.\boldsymbol{w}^{H} \boldsymbol{C}_{k, n}^{H} \hat{\boldsymbol{v}}_{k l}^{(m)}(p)\right|^{2} \leq \gamma_{k}^{2}\right\}
\end{array}
$$

In the OBE methodology, the feasibility set is sought by successively refining membership sets $\mathcal{W}_{k}(p)$ defined at time $p$ as

$$
\begin{aligned}
\mathcal{W}_{k}(p)=\bigcap_{r=0}^{p}\left\{w \in \mathcal{C}^{N-1}: \sum_{m=0}^{M-1} \mid \boldsymbol{w}_{k, q}^{H} \hat{\boldsymbol{v}}_{k l}^{(m)}(p)\right. \\
\left.-\left.\boldsymbol{w}^{H} C_{k, n}^{H} \hat{\boldsymbol{v}}_{k l}^{(m)}(p)\right|^{2} \leq \gamma_{k}^{2}\right\}
\end{aligned}
$$

However, the complexity in exactly computing $\mathcal{W}_{k}(p)$ is overwhelming even for small $N$ and $p$. OBE algorithms circumvent this problem by recursively updating hyper-ellipsoids $\mathcal{E}_{k}(p)$ which tightly outerbound the membership sets $\mathcal{W}_{k}(p)$ for all $p$ (Fig. 2). It follows that $\mathcal{E}_{k}(p)$ also outerbounds $\Theta_{k}\left(N, \gamma_{k}\right)$ at all times, since $\Theta_{k}\left(N, \gamma_{k}\right)$ is a subset of $\mathcal{W}_{k}(p)$, i.e.,. $\mathcal{E}_{k}(p) \supset \mathcal{W}_{k}(p) \supset \Theta_{k}\left(N, \gamma_{k}\right)$ for all $p$. Thus a recursive formulation can be used to update $\mathcal{E}_{k}(p)$ with each incoming chip vector at the symbol rate. Equation (3.3) can be rewritten in matrix form for the array as

$$
\boldsymbol{e}_{k l}^{*}(p)=\boldsymbol{U}_{k, q}(p)-V_{k, n}^{H}(p) \boldsymbol{w}_{k, a}
$$

where

$$
\begin{aligned}
& \boldsymbol{V}_{k, q}(p) \triangleq\left[\hat{\boldsymbol{v}}_{k l}^{(0)}(p), \hat{\boldsymbol{v}}_{k l}^{(1)}(p), \cdots \hat{\boldsymbol{v}}_{k l}^{(M-1)}(p)\right] \\
& \boldsymbol{U}_{k, q}(p) \triangleq \boldsymbol{V}_{k, q}^{H}(p) \boldsymbol{w}_{k, q} \\
& \text { and } \\
& \boldsymbol{V}_{k, n}(p) \triangleq\left[\boldsymbol{C}_{k, n}^{H} \hat{\boldsymbol{v}}_{k l}^{(0)}(p), \boldsymbol{C}_{k, n}^{H} \hat{\boldsymbol{v}}_{k l}^{(1)}(p), \cdots, \boldsymbol{C}_{k, n}^{H} \hat{\boldsymbol{v}}_{k l}^{(M-1)}(p)\right] .
\end{aligned}
$$




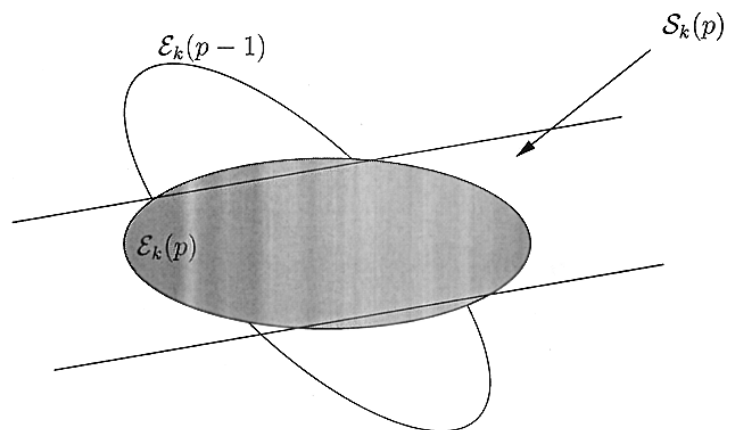

Fig. 2. Schematic depicting operation of OBE recursions in two dimensions $(N=3$ and $M=1)$.

Then as per the set-membership framework, let the error specification for the $k$ th user be

$$
\left\|\boldsymbol{e}_{k l}(p)\right\|^{2} \leq \gamma_{k}^{2}
$$

where $\|\cdot\|$ denotes the vector $l_{2}$ norm and $\gamma_{k}^{2}$ is an appropriately chosen constant. The selection of $\gamma_{k}^{2}$ is addressed later in this section. Define $\mathcal{S}_{k}(p)$ as a degenerate ellipsoid as

$$
\mathcal{S}_{k}(p)=\left\{\boldsymbol{w} \in \mathcal{C}^{N-1}:\left\|\boldsymbol{U}_{k, q}(p)-V_{k, n}^{H}(p) \boldsymbol{w}\right\|^{2} \leq \gamma_{k}^{2}\right\} .
$$

Let the membership set at time $(p-1)$ be given by

$$
\begin{aligned}
\mathcal{E}_{k}(p-1)=\{ & \left\{\boldsymbol{w} \in \mathcal{C}^{N-1}:\left[\boldsymbol{w}-\boldsymbol{w}_{k}(p-1)\right]^{H}\right. \\
& \left.\cdot \boldsymbol{P}_{k}^{-1}(p-1)\left[\boldsymbol{w}-\boldsymbol{w}_{k}(p-1)\right] \leq \sigma_{k}^{2}(p-1)\right\}
\end{aligned}
$$

where $\boldsymbol{P}_{k}(p-1)$ is a symmetric positive-definite matrix and $\boldsymbol{w}_{k}(p-1)$ is the center of the ellipsoid. An ellipsoid that contains $\mathcal{E}_{k}(p-1) \cap \mathcal{S}_{k}(p)$ is given by

$$
\begin{aligned}
\mathcal{E}_{k}(p)=\{ & \left\{\boldsymbol{w} \in \mathcal{C}^{N-1}:\left[1-\lambda_{k}(p)\right]\left[\boldsymbol{w}-\boldsymbol{w}_{k}(p-1)\right]^{H}\right. \\
& \cdot \boldsymbol{P}_{k}^{-1}(p-1)\left[\boldsymbol{w}-\boldsymbol{w}_{k}(p-1)\right] \\
& +\lambda_{k}(p)\left\|\boldsymbol{U}_{k, q}(p)-\boldsymbol{V}_{k, n}^{H} \boldsymbol{w}\right\|^{2} \\
\leq & {\left.\left[1-\lambda_{k}(p)\right] \sigma_{k}^{2}(p-1)+\lambda_{k}(p) \gamma_{k}^{2}\right\} }
\end{aligned}
$$

where $\lambda_{k}(p)$ is a real number in $(0,1)$. It can now be shown that there exists a symmetric positive-definite $\boldsymbol{P}_{k}(p)$ and a positive scalar $\sigma_{k}^{2}(p)$ such that

$$
\begin{aligned}
& \mathcal{E}_{k}(p)=\left\{\boldsymbol{w} \in \mathcal{C}^{N-1}:\left[\boldsymbol{w}-\boldsymbol{w}_{k}(p)\right]^{H}\right. \\
&\left.\cdot \boldsymbol{P}_{k}^{-1}(p)\left[\boldsymbol{w}-w_{k}(p)\right] \leq \sigma_{k}^{2}(p)\right\}
\end{aligned}
$$

is a well-defined ellipsoid (Fig. 2).
Proposition 3.1: Consider the inequalities (3.11) and (3.12) above. Define

$$
\begin{aligned}
& \boldsymbol{d}_{k}(p) \triangleq \boldsymbol{U}_{k, q}(p)-V_{k, n}^{H}(p) \boldsymbol{w}_{k}(p-1) \\
& \boldsymbol{G}_{k}(p) \triangleq \boldsymbol{V}_{k, n}^{H}(p) \boldsymbol{P}_{k}(p-1) \boldsymbol{V}_{k, n}(p)
\end{aligned}
$$

and

$$
\boldsymbol{Q}_{k}(p) \triangleq\left[1-\lambda_{k}(p)\right] \boldsymbol{I}_{M}+\lambda_{k}(p) \boldsymbol{G}_{k}(p) .
$$

The following recursive update equations may be obtained:

$$
\begin{gathered}
\boldsymbol{P}_{k}(p)=\frac{1}{1-\lambda_{k}(p)}\left[\boldsymbol{P}_{k}(p-1)-\lambda_{k}(p) \boldsymbol{P}_{k}(p-1)\right. \\
\left.\cdot \boldsymbol{V}_{k, n}(p) \boldsymbol{Q}_{k}^{-1}(p) \boldsymbol{V}_{k, n}^{H}(p) \boldsymbol{P}_{k}(p-1)\right] \\
\boldsymbol{w}_{k}(p)=\boldsymbol{w}_{k}(p-1)+\lambda_{k}(p) \boldsymbol{P}_{k}(p) \boldsymbol{V}_{k, n}(p) \boldsymbol{d}_{k}(p)
\end{gathered}
$$

and

$$
\begin{aligned}
\sigma_{k}^{2}(p)= & {\left[1-\lambda_{k}(p)\right] \sigma_{k}^{2}(p-1)+\lambda_{k}(p) \gamma_{k}^{2} } \\
& -\left[1-\lambda_{k}(p)\right] \lambda_{k}(p) \boldsymbol{d}_{k}^{H}(p) \boldsymbol{Q}_{k}^{-1}(p) \boldsymbol{d}_{k}(p) .
\end{aligned}
$$

The last three equations in Proposition 3.1 constitute the recursions of the OBE algorithm. In order to compute the optimal update factor the parameter $\sigma_{k}^{2}(p)$ is minimized. $\sigma_{k}^{2}(p)$ can be considered to be a bound on the estimation error at the $p$ th step and is closely related to other popular measures of optimization such as volume and trace of the bounding ellipsoid [5], [15]. Unlike these measures, minimization of $\sigma_{k}^{2}(p)$ lends itself to a very efficient test for innovation. A tight upper bound on $\sigma_{k}^{2}(p)$, denoted by $\sigma^{\prime 2}(p)$, is given by

$$
\begin{aligned}
\sigma^{\prime 2}(p)= & {\left[1-\lambda_{k}(p)\right] \sigma_{k}^{2}(p-1)+\lambda_{k}(p) \gamma_{k}^{2} } \\
& -\left[1-\lambda_{k}(p)\right] \lambda_{k}(p) \frac{\boldsymbol{d}_{k}^{H}(p) \boldsymbol{d}_{k}(p)}{\left[1-\lambda_{k}(p)\right]+\lambda_{k}(p) g_{k}(p)}
\end{aligned}
$$

where $g_{k}(p)=\left\|\boldsymbol{G}_{k}(p)\right\|$. Denote the optimal $\lambda_{k}(p)$ by $\lambda_{k}^{o}(p)$ (which lies in $\left[0, \alpha_{k}\right]$ for some real scalar design parameter $\left.\alpha_{k} \in(0,1)\right)$ and define the quantity

$$
\beta_{k}(p) \triangleq \frac{\gamma_{k}^{2}-\sigma_{k}^{2}(p-1)}{\boldsymbol{d}_{k}^{H}(p) \boldsymbol{d}_{k}(p)}
$$

Proposition 3.2: Minimization of $\sigma^{\prime 2}(p)$ with respect to $\lambda_{k}(p)$ leads to the following update condition:

1) if $\beta_{k}(p) \geq 1$, then $\lambda_{k}^{o}(p)=0$;

2) otherwise $\lambda_{k}^{o}(p)=\min \left(\alpha_{k}, \nu_{k}(p)\right)$ where $\nu_{k}(p)$ takes the values shown at the bottom of this page.

See [15] for proofs of Propositions 3.1 and 3.2. This result is used for computing the optimal update parameter $\lambda_{k}^{o}(p)$ at each step. Equation (3.16) and Proposition 3.2 constitute the condition for data selectivity or no-update. At any time $p$, the center of the ellipsoid $w_{k}(p)$ is taken as an estimate of the

$$
\nu_{k}(p)= \begin{cases}\alpha_{k}, & \text { if } \boldsymbol{d}_{k}^{H}(p) \boldsymbol{d}_{k}(p)=0 \\ \frac{\left(1-\beta_{k}(p)\right)}{2}, & \text { if } g_{k}(p)=1 \\ \frac{1}{1-g_{k}(p)}\left[1-\sqrt{\left.\frac{g_{k}(p)}{1+\beta_{k}(p)\left[g_{k}(p)-1\right]}\right],},\right. & \text { if } 1+\beta_{k}(p)\left[g_{k}(p)-1\right]>0 \\ \alpha_{k}, & \text { if } 1+\beta_{k}(p)\left[g_{k}(p)-1\right] \leq 0\end{cases}
$$


adaptive component of the detector in (3.1). To initialize the algorithm, $\mathcal{E}_{k}(0)$ is chosen such that

$$
\mathcal{E}_{k}(0)=\left\{\boldsymbol{w} \in \mathcal{C}^{N-1}:\|\boldsymbol{w}\|^{2} \leq 1 / \mu\right\}
$$

where $\mu \ll 1$ resulting in $\mathcal{E}_{k}(0)$ being a suitably large initial hyper-sphere. In other words, the following initial values may be chosen:

$$
\boldsymbol{P}_{k}(0)=\boldsymbol{I}_{N-1}, \quad \boldsymbol{w}_{k}(0)=\mathbf{0}, \quad \text { and } \quad \sigma_{k}^{2}(0)=1 / \mu \text {. }
$$

In (3.5) and the subsequent treatment in this section, an explicit dependence of $\gamma_{k}^{2}$ on time (i.e., on $p$ ) is not shown. However, in general, $\gamma_{k}^{2}$ is not necessarily constant over time. Since one of the goals of using a multiuser detector is to simplify power control mechanisms, a time-varying $\gamma_{k}^{2}$ error specification is beneficial to the adaptive detector's performance in fading channels. Assuming that channel fading estimates are available, the error specification is appropriately modified to reflect the received power estimate of the desired user (see Section V). Such estimates are typically obtained via the use of pilot symbols which are periodically inserted in a framing pattern or via dedicated pilot channels. Also, such estimates are required by RAKE combiner (except when simple selection diversity is used). The next stage of processing in the receiver of Fig. 1 entails the combining of the detector outputs corresponding to each RAKE branch from all array elements via a bank of beamformers.

\section{DOA ESTIMATION AND BEAMFORMING}

Beamforming is carried out based on DOA estimates of the desired users' signals. This approach is particularly well suited for DS-CDMA systems in which there are a large number of spatially distributed interferers. As depicted in Fig. 1, in a frequency-selective multipath environment, the outputs of each RAKE branch from each sensor in a uniform linear array are fed into a bank of beamformers. Since steering vectors are computed from DOA estimates, array calibration is required in this approach. Each beamformer strives to be spatially selective in the direction of the particular RAKE branch output and requires DOA estimates of the desired users' signals at the output of each branch for computing its coefficients.

Carrying out DOA estimation prior to despreading can be a formidable task. Most subspace-based methods are not applicable due to the large number of independent signals and typically small number of array elements. Postdetection DOA estimation, on the other hand, is a viable option. Several methods have been proposed in the literature, including subspacebased methods such as Weighted Subspace Fitting [2], ESPRIT [25], and an eigendecomposition based method [21]. The iterative ML algorithm based on alternating projections proposed in [31] is also applicable. The structure of a DS-CDMA system, however, allows for a much simpler and robust approach. This section describes an approximate Maximum-Likelihood DOA Estimator (DOA-MLE) used for beamforming. In addition to inheriting the desirable properties of MLE's, it also turns out to be simple and intuitive. The basic idea is to partition the array into groups of two consecutive sensors or doublets. The algorithm then exploits the fading correlation between closely spaced doublet elements to extract the spatially induced phase differences between the postcorrelation complex baseband outputs. Each doublet operates independently to compute the DOA-MLE and the estimates from multiple doublets are then suitably combined.

\section{A. Maximum-Likelihood DOA Estimation}

The relationship between angle of arrival, beamwidth of arriving signals, and antenna spacing has been explored in [26] and the references therein. For an interelement spacing of $\rho$ and narrowband signal wavelength $v$, the fading experienced at adjacent sensors is almost perfectly correlated for small values of $\rho / v$ (such as 0.5 or less) and angle spread $\triangle$ (such as $\Delta<10^{\circ}$ ). Thus interelement spacing $\rho$ for each doublet is assumed to be such that the two sensors experience nearly identical fading. Consider sensor $m$ and $(m+1)$ constituting a doublet. Using (2.9) and (3.1)

$$
\begin{aligned}
e_{k l l}^{(m)}(p) & =\boldsymbol{w}_{k, q}^{H} \hat{\boldsymbol{v}}_{k l}^{(m)}(p)-\boldsymbol{w}_{k}^{H}(p) \boldsymbol{C}_{k, n}^{H} \hat{\boldsymbol{v}}_{k l}^{(m)}(p) \\
& =\sqrt{P_{k}} a_{k l}^{(m)} A_{k}(p)+\left[\left(\boldsymbol{v}_{k}^{H} / N\right)-\boldsymbol{w}_{k}^{H}(p) \boldsymbol{C}_{k, n}^{H}\right] I_{k l}^{(m)}(p) \\
& =\sqrt{P_{k}} a_{k l}^{(m)} A_{k}(p)+\tilde{I}_{k l}^{(m)}(p)
\end{aligned}
$$

where $\boldsymbol{w}_{k}(p)$ denotes the updated weight vector from the recursions of Proposition $3.1 ; I_{k l}^{(m)}(p)$ denotes the collective interference terms in (2.7); and $\widetilde{I}_{k l}^{(m)}(p)$ is appropriately defined. Given $P$ observations from time instants $(p-P+1)$ to $p$, an approximate MLE of the spatial phase difference can be obtained straightforwardly (see Appendix B) as

$$
\hat{\phi}_{k l}^{(m)}(p)=\tan ^{-1}\left[\frac{\operatorname{Im}\left[\sum_{r=p-P+1}^{p} e_{k l}^{(m+1)}(r)\left(e_{k l}^{(m)}(r)\right)^{*}\right]}{\operatorname{Re}\left[\sum_{r=p-P+1}^{p} e_{k l}^{(m+1)}(r)\left(e_{k l}^{(m)}(r)\right)^{*}\right]}\right]
$$

where $\left(e_{k l}^{(m)}(r)\right)^{*}$ denotes the complex conjugate of $e_{k l}^{(m)}(r)$. Conceptually similar approaches have also been used for frequency estimation where the corresponding problem is converted to a phase estimation problem. At every time instant, each doublet contributes a DOA-MLE for each user being tracked. Since all array elements are used for beamforming, the DOA-MLE's from each doublet are combined to form a single estimate. The simplest method is to average the outputs from each doublet, while other schemes may be readily conceived. For instance, a suitable selection criterion can be adopted for selecting the "best" DOA-MLE from among the doublets. In any case, denote the final DOA estimate at time $p$ by $\hat{\phi}_{k l}(p)$. Let the $l$ th-beamformer weight vector be denoted by $\boldsymbol{b}_{k l l}$ (see Fig. 1); the data covariance matrix at the output of the $l$ th RAKE branch by $\boldsymbol{R}_{l}$; and the steering vector for the $k$ th-user's $l$ th path be given by

$$
\boldsymbol{s}_{k l}(p)=\left[1, e^{j \hat{\phi}_{k l}^{(1)}(p)}, \cdots, e^{j \hat{\phi}_{k l}^{(M-1)}(p)}\right]^{H} .
$$

Adopting the classical minimum variance distortionless response (MVDR) criterion for computing the beamformer weights [28]

$$
\boldsymbol{b}_{k l l}(p)=\frac{\boldsymbol{R}_{l}^{-1} \boldsymbol{s}_{k l l}(p)}{\boldsymbol{s}_{k l l}^{H}(p) \boldsymbol{R}_{l}^{-1} \boldsymbol{s}_{k l}(p)}
$$


The output of the $l$ th beamformer is then given by

$$
y_{k l}(p)=\boldsymbol{b}_{k l l}^{H}(p) \boldsymbol{e}_{k l}(p) .
$$

$\boldsymbol{b}_{k l}(p)$ is typically block-updated using (4.4) above at a rate commensurate with the available processing power while $\boldsymbol{R}_{l}$ is approximated by a suitably windowed temporal average. Clearly, this method of obtaining the weights for the bank of beamformers is not optimized for implementation as such but this issue has been extensively studied in the literature, see, e.g., [28]. However, the problem is alleviated by the fact that the dimensionality of $\boldsymbol{b}_{k l}$ is not large due to typical values of $M \ll N$. Experience has shown that block weight updating with a reasonably chosen temporal block size (instead of continuous recursive updates) for postdetection beamformers is a reasonable tradeoff for most channels and interference scenarios and is used in this paper. Other beamforming methods, such as the maximum SINR method [28], may also be used. The length of the temporal window $P$ used by the DOAMLE algorithm can be adjusted according to the expected rate of change of the DOA of incoming signals from the desired user. For instance, this would depend on mobile speeds, cell geometry, and symbol rate.

\section{B. Performance Analysis}

Compared to conventional eigendecomposition-based methods [25], the simplicity of the proposed method is apparent. Only simple arithmetic operations and functional table lookup are required. In formulating the DOA-MLE, all multipath components delayed by more than one chip time appear as postdecorrelation additive noise. The DOA-MLE can be computed independently for all users being demodulated and this ensures that there are no "resolution" problems, as is the case when DOA estimates for multiple sources are simultaneously being computed. For $M$ sensor elements and $K$ DOA's being estimated, the DOA-MLE requires $\mathcal{O}(M K)$ operations and can be used for all sources being demodulated with one or more doublets. Consider the argument of the inverse tangent function in (4.2) and let

$$
c_{k l}^{(m)}(p) \triangleq \frac{1}{P} \sum_{r=p-P+1}^{p} e_{k l}^{(m+1)}(r)\left(e_{k l}^{(m)}(r)\right)^{*} .
$$

Using (4.1), (B.3), and the uncorrelatedness of the transmitted symbols

$$
\begin{aligned}
E\left[c_{k l}^{(m)}(p)\right] & =\frac{P_{k}}{P} \sum_{r=p-P+1}^{p} E\left[a_{k l l}^{(m+1)}\left(a_{k l}^{(m)}\right)^{*}\right] \\
& =\frac{P_{k}}{P} \sum_{r=p-P+1}^{p} E\left[\left|a_{k l}^{(m)}\right|^{2} e^{j \phi_{k l}^{(1)}}+\epsilon_{k l}^{(m+1)}\left(a_{k l}^{(m)}\right)^{*}\right] \\
& =\frac{P_{k}}{P} e^{j \phi_{k l}^{(1)}} \sum_{r=p-P+1}^{p} E\left[\left|a_{k l}^{(m)}\right|^{2}\right] \\
& =e^{j \phi_{k l}^{(1)}} P_{k} E\left[\left|a_{k l}^{(m)}\right|^{2}\right]
\end{aligned}
$$

where $\epsilon_{k l l}^{(m)}$ is assumed to be zero-mean and uncorrelated. Thus assuming a second-order stationary fading process, the estimator for $\tan \left(\phi_{k l}^{(1)}\right)$ is unbiased. Similarly, taking the second moment

$$
\begin{aligned}
E\left[\left|c_{k l}^{(m)}(p)\right|^{2}\right]= & \frac{P_{k}^{2}}{P^{2}} \sum_{r=p-P+1}^{p} E\left[\mid e^{j \phi_{k l}^{(1)}\left|a_{k l}^{(m)}\right|^{2}}\right. \\
& \left.+\left.\epsilon_{k l l}^{(m+1)}\left(a_{k l}^{(m)}\right)^{*}\right|^{2}\right] \\
= & \frac{P_{k}^{2}}{P^{2}} \sum_{r=p-P+1}^{p} E\left[\left|a_{k l}^{(m)}\right|^{4}\right] \\
& +E\left[\left|\epsilon_{k l}^{(m+1)}\right|^{2}\left|a_{k l}^{(m)}\right|^{2}\right] \\
= & \frac{P_{k}^{2}}{P} E\left[\left|a_{k l}^{(m)}\right|^{4}\right]+E\left[\left|\epsilon_{k l}^{(m+1)}\right|^{2}\left|a_{k l}^{(m)}\right|^{2}\right]
\end{aligned}
$$

Again, assuming stationarity up to the fourth-order of the fading process, the estimate for $\tan \left(\phi_{k l}^{(1)}\right)$ is asymptotically consistent. Thus two approximations are made in estimating $\phi_{k: l}^{(1)}$-due to angle spreading and due to the inverse tangent operation.

\section{RAKE Reception}

Consider now the final stage of the adaptive array receiver, just prior to the slicer. The $L \times 1$ vector output $\boldsymbol{y}_{k}(p)=$ $\left[y_{k_{0}}(p), y_{k_{1}}(p), \cdots, y_{k(L-1)}(p)\right]^{T}$ of the bank of beamformers is fed into the RAKE combiner to obtain symbol estimates for each desired user. Denote the $k$ th users' RAKE combining coefficients by $\boldsymbol{r}_{k}(p)=\left[r_{k 0}(p), r_{k 1}(p), \cdots, r_{k(L-1)}(p)\right]^{T}$. The output of the $k$ th-users' combiner is then given by

$$
\hat{A}_{k}(p)=r_{k}^{H}(p) \boldsymbol{y}_{k}(p) .
$$

There are several RAKE combining algorithms, the classical ones being maximal ratio, equal gain, and selection combining [14]. Any one of these methods or other variations in the literature [32] may be used. Such methods typically hinge on the slowly fading assumption and make use of special pilot symbols or training sequences to update the RAKE combining coefficients. In a flat fading environment, the RAKE combining reduces to a single 1-tap equalizer yielding

$$
\hat{A}_{k}(p)=r_{k 0}(p) y_{k}(p) .
$$

The equalizer operates on the received complex baseband symbol before slicing by correcting the magnitude and phase of the single beamformer's symbol rate output.

\section{Simulation Results}

Consider first the performance of the proposed receiver in a flat Rayleigh fading environment. Fading coefficients were generated using the standard Jakes model [14] with a normalized Doppler bandwidth of 0.001. Fig. 3 depicts the ensemble averaged signal-to-interference ratio (SIR) obtained using a nonadaptive MF and an adaptive multiuser detector with OBE, standard LMS, and RLS algorithms. The latter three algorithms are used for updating the GSC's $(N-1) \times 1$ adaptive weight vector component with a single signal-preserving constraint. Coherent quadrature modulation is used with a processing gain of $N=16$ with a single antenna element $(M=1)$ and a background SNR of $20 \mathrm{~dB}$ due to the additive white 


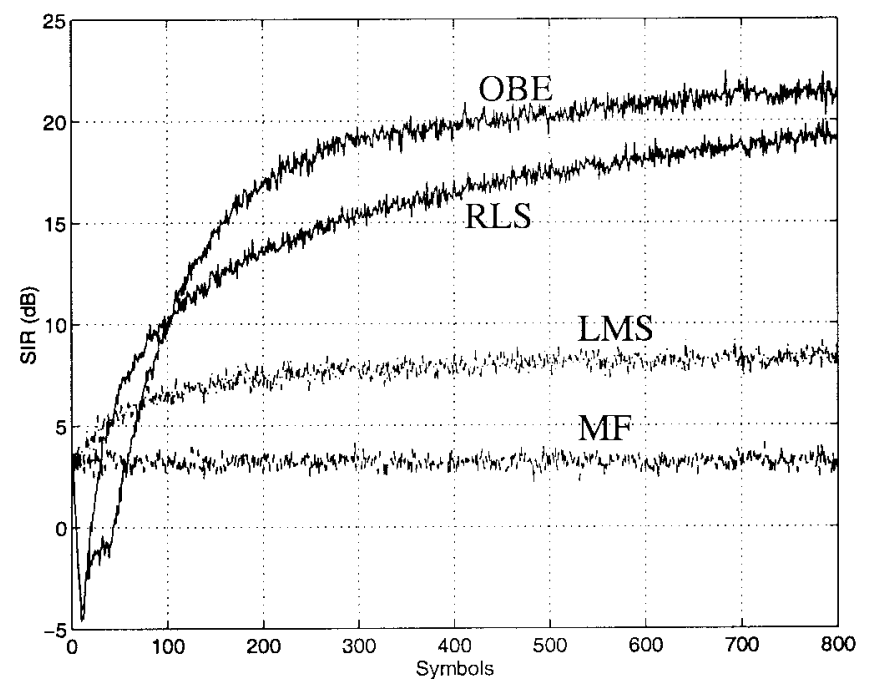

Fig. 3. SIR using MF, OBE, and RLS algorithms. Processing gain $N=16$, $M=1$.

Gaussian noise (AWGN). Similar curves are obtained for larger processing gains. There are a total of 11 active users including the desired user, each transmitting with equal power. This represents a severe interference environment in which the MF detector cannot provide adequate performance. The LMS update gain is fixed at 0.001 while the RLS forgetting factor is chosen to be 0.99. Larger values of the LMS update factor resulted in slightly faster convergence. However, they also resulted in frequent divergence of the weight vector and were therefore not used. The value of $\alpha_{0}$ was chosen to be 0.2 for all simulations. The error specification $\left(\gamma_{k}^{2}\right)$ for OBE is set at $M \hat{P}_{0}(p)+\xi$ where $\hat{P}_{0}(p)$ is an estimate of the desired user's received power and $\xi$ is design parameter chosen to be unity. The SIR at the $p$ th symbol is calculated according to

$$
\operatorname{SIR}(p)=\frac{\sum_{r}\left|w_{r}^{H}(p) v_{\mathrm{sig}, r}(p)\right|^{2}}{\sum_{r}\left|w_{r}^{H}(p) v_{\mathrm{int}, r}(p)\right|^{2}}
$$

where the ensemble average is carried out over 200 independent trials in each of which the signature sequences are generated randomly; $w_{r}(p)$ denotes the detector weight vector in the $n$th trial at the $p$ th symbol; $\boldsymbol{v}_{\mathrm{sig}, r}(p)$ and $\boldsymbol{v}_{\mathrm{int}, r}(p)$ denote the $N \times 1$ received signal and interference chip vectors in the $n$th trial at the $p$ th symbol, respectively. $w$ is adapted using OBE, LMS, and RLS or is fixed when the MF is used.

Using the complexity reduction technique described in [6], with an update rate of about $20 \%$, the average computational load of OBE versus RLS over a block of, say, 500 symbols is about one-fifth. Note that this gain can be smaller for the time period when a user initiates communication and rapid parameter updates occur and higher in steady state. Using the statistical time-shared updator method proposed in [10], and assuming 64 maximum users at the base station with typical cellular traffic characteristics, approximately only nine update processors are required [10], i.e., a seven-fold reduction in hardware complexity for updating the detector vectors. To realize this benefit, the base-station architecture must be able to

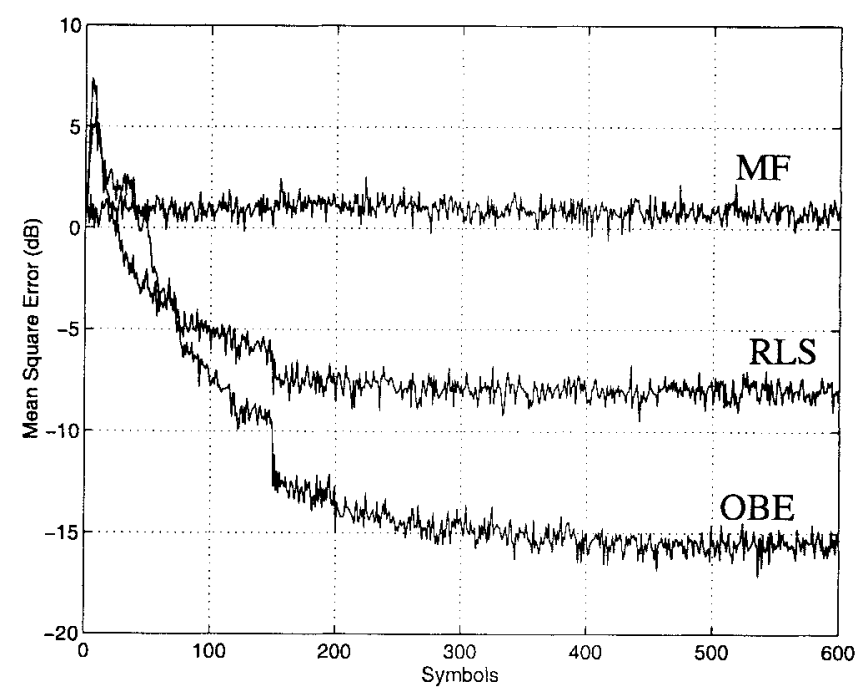

Fig. 4. MSE using MF, OBE, and RLS algorithms. Processing gain $N=16$, $M=4$.

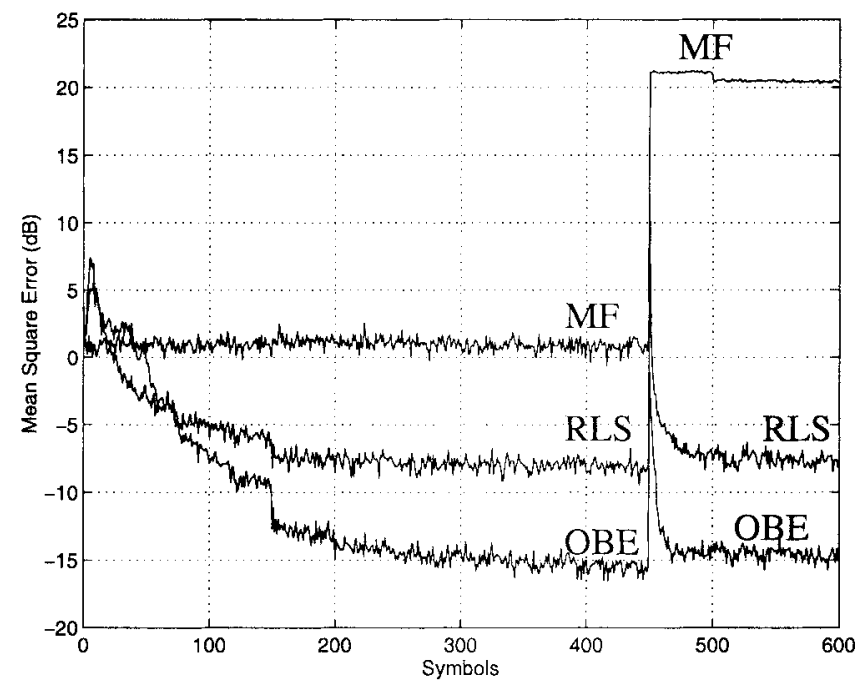

Fig. 5. MSE using MF, OBE, and RLS algorithms with strong interferer. Processing gain $N=16, M=4$.

allow sharing of baseband signal processing resources among multiple channels.

An antenna array with $M=4$ elements is used in Fig. 4 and the ensemble averaged mean-squared error (MSE) at the receiver output is computed. Using (3.4), the nonadaptive MMSE for these parameters is $-16 \mathrm{~dB}$. It is assumed that channel estimates are available at the receiver to equalize the preslicer symbol rate samples. With a forgetting factor equal to one, the RLS algorithm also achieved the MMSE bound. Some degradation in the achievable MSE is to be expected when the channel estimates are not perfect. The tracking behavior of the OBE detector is compared with an RLS detector in Fig. 5. The nonadaptive MF is also shown for comparison. It is seen that both the OBE and RLS algorithms adapt to the strong interferer introduced at the 450th symbol. The OBE detector settles down in about 25-50 symbols while the RLS detector takes about 75-100 symbols. The DOA's of all 11 users are randomly distributed in the range $\left[-60^{\circ},+60^{\circ}\right]$, each with an angle 

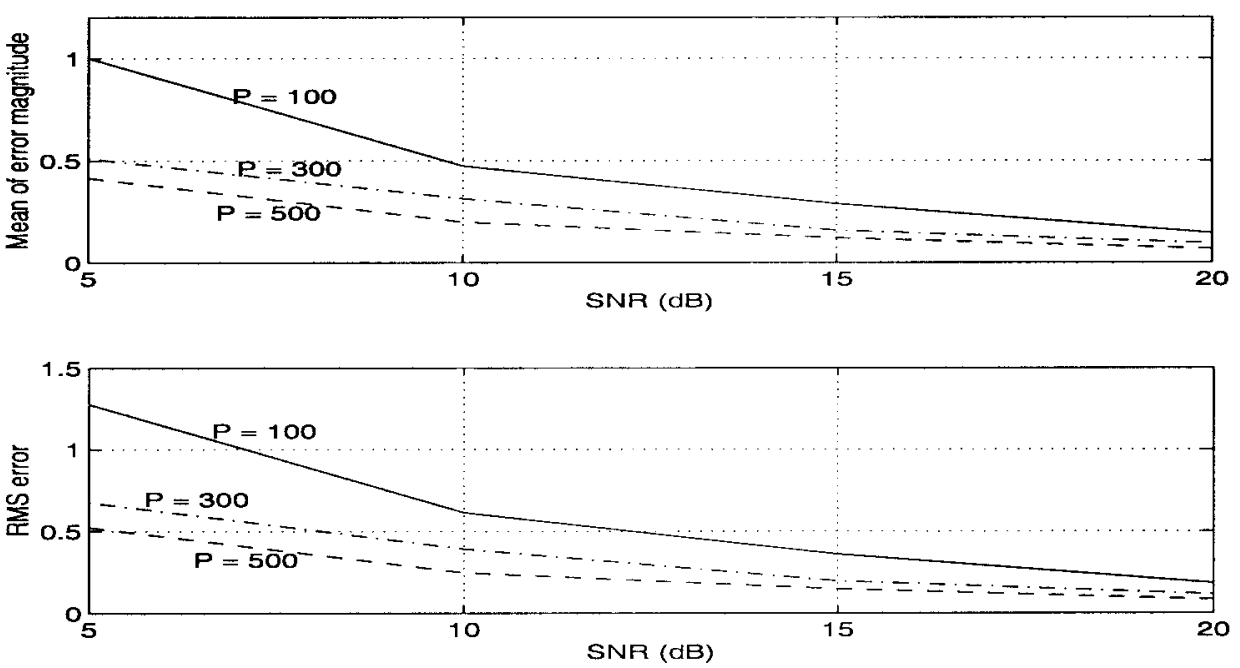

Fig. 6. Magnitude and rms value of DOA estimation error in degrees using DOA-MLE algorithm: number of symbols $P$ versus SNR for angle spread $\triangle=0$.
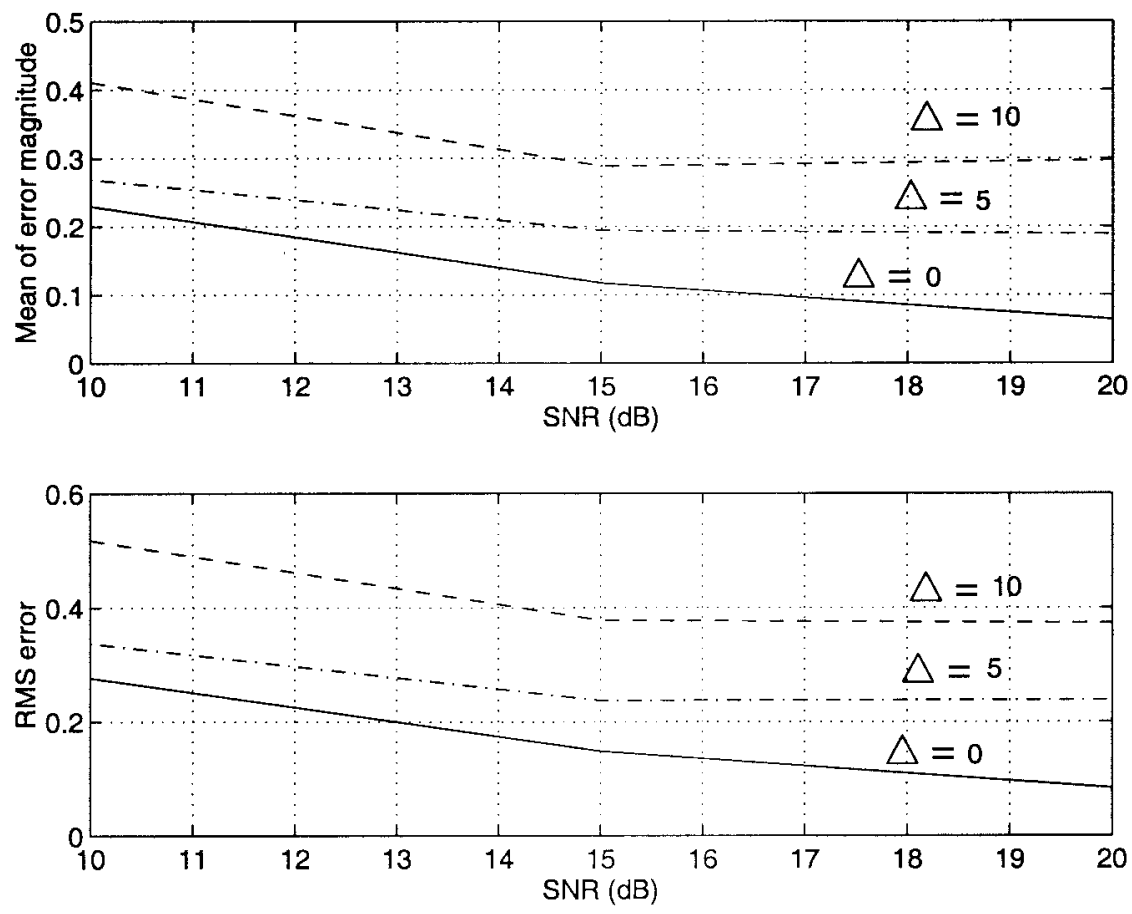

Fig. 7. Magnitude and rms value of DOA estimation error in degrees using DOA-MLE algorithm: angle spread $\triangle$ versus SNR for number of symbols $P=500$.

spreading of $5^{\circ}$. An intersensor spacing of $\rho=v / 8$ is used. Perfect correlation is assumed between the fading experienced at adjacent sensors. This is known to be a good approximation for the sensor spacing and angle spreads under consideration [26]. The DOA-MLE algorithm (see (4.2)) is used for DOA estimation. Estimates from multiple doublets are averaged at each update instant. Beamformer weights are obtained using the MVDR criterion (see (4.4)]). The beamformer weights are initialized to have an omnidirectional response, the first update is made at symbol 150, and thereafter every 50 symbols using a sliding temporal window to update the DOA-MLE.

Figs. 6 and 7 show the performance of the DOA-MLE algorithm alone. The magnitude and root-mean-square (rms) value of the estimation error under different postdetection SNR ratios are shown. Each data point is obtained by ensemble averaging over 1000 independent trials using a single sensor doublet with spacing $\rho=v / 8$. In Fig. 6, the angle spread $\triangle$ is held fixed at $0^{\circ}$ while the number of symbols $P$ used for forming the estimate is varied. In Fig. 7, $P=500$ while $\triangle$ is varied. The angle spread is assumed to arise from multipath subcomponents uniformly distributed in the interval $[-\triangle / 2, \triangle / 2]$. Fig. 8 depicts the signal-to-noise-plusinterference ratio (SINR) using RLS and OBE detectors after 750 symbols versus the number of array elements for $N=$ 16 with a single RAKE path $(L=1)$. For each case, there are ten interferers as before. Fig. 9 shows the effect of increasing the power of interferers relative to the desired signal power using $N=16$ and $M=2$. The resulting SINR is 


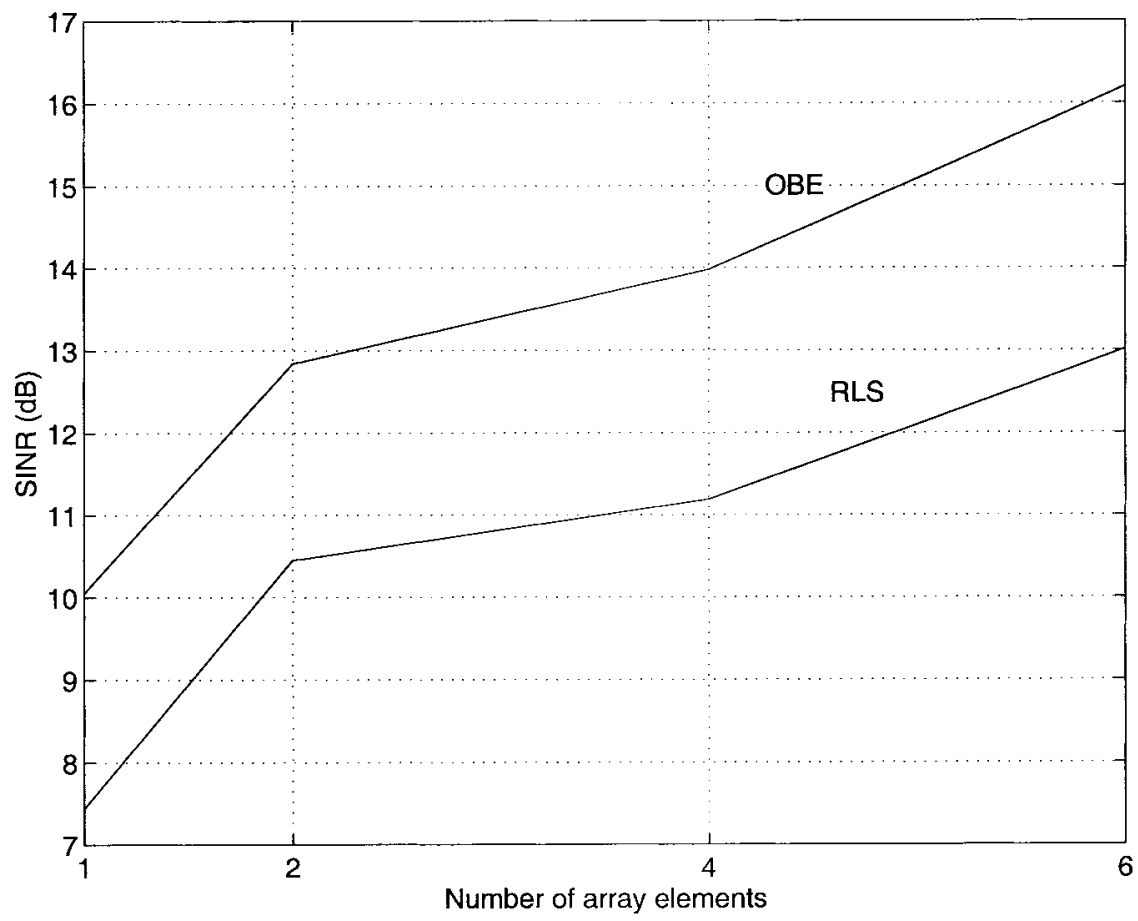

Fig. 8. SINR using OBE and RLS detectors versus number of array elements. Total of ten equal power interferers with same power as desired user. Processing gain $N=16$.

computed after 750 symbols of adaptation. The performance of OBE and RLS is compared in a frequency-selective Rayleigh fading environment while keeping the same DOA estimation, beamforming and RAKE combining in the remaining receiver stages. The ensemble averaged SINR at the $p$ th symbol is computed at the output of each RAKE branch as

$$
\operatorname{SINR}(p)=\frac{\sum_{r}\left|\boldsymbol{b}_{r}^{H}(p) \boldsymbol{V}_{\mathrm{sig}, r}^{H}(p) \boldsymbol{w}_{r}(p)\right|^{2}}{\sum_{r}\left|\boldsymbol{b}_{r}^{H}(p)\left(\boldsymbol{V}_{\mathrm{int}, r}(p)+N_{r}(p)\right) \boldsymbol{w}_{r}(p)\right|^{2}}
$$

where $\boldsymbol{V}_{\mathrm{sig}, r}$ and $\boldsymbol{V}_{\mathrm{int}, r}$ denote $N \times M$ signal and interference matrices, each column of which corresponds to the received signal and interference chip vector during the $p$ th symbol at different array elements; $\boldsymbol{N}_{r}(p)$ denotes the matrix of additive noise samples in the same fashion and $\boldsymbol{b}_{r}(p)$ denotes the beamformer weight vector. When multiple RAKE branches are used, the SINR at the output of each branch is defined in the same manner. In Fig. 10, the probability of bit error $\left(P_{b}\right)$ is calculated using the SINR after 750 symbols with $N=32, K=10$ interfering users, each contributing 2 RAKE branches. $P_{b}$ is computed for coherent quadrature modulation with $L$ RAKE branches as [23]

$$
P_{b} \approx\left(\frac{1}{4 \cdot \operatorname{SINR}}\right)^{L}\left(\begin{array}{c}
2 L-1 \\
L
\end{array}\right)
$$

where the SINR is measured at each RAKE branch. Also, a constant power profile is assumed across RAKE branches and maximal ratio combining is used.

\section{A. Discussion}

In the simulations results described above, it is observed that the OBE algorithm outperforms the RLS algorithm in terms of convergence and complexity. While OBE and RLS update equations bear a striking resemblance, this difference in performance can be primarily attributed to the datadependent optimization of the OBE update factor at each symbol. The performance of RLS is sensitive to the value of the forgetting factor while the OBE methodology provides a natural and convenient way for optimizing the update factor. A detailed comparison of the tracking properties of OBE versus RLS appears in [24]. This optimization also leads to sparse updates which have been utilized for significant computational savings [6], [10]. The rate of convergence assumes particular importance when uplink transmission takes place in a framing structure with periodic training symbols. In such cases, which are typical, the adaptive algorithms are required to rapidly converge and provide parameter estimates until they are "reinitialized" in the next frame. Overall, the percentage of OBE updates is $20 \%$ or less and far lower when the percentage of updates is computed over a larger number of symbols.

The multiuser detector is operated with a single constraint above. If desired, a decrease in the number of adaptive weights can be achieved in the GSC framework by additional constraints. This will also lead to faster suppression of intracell interferers if their timing and signature sequences are known. However, a drawback of imposing additional constraints is that it reduces the degrees of freedom available to suppress sudden interferers. In any case, active cochannel users from neighboring cells are known to be a significant and unpredictable source of interference, making it hard to construct appropriate constraints in advance.

Relative to OBE and RLS, the LMS algorithm does not perform adequately $-a$ finding consistent with that in the literature [18]. In situations where use of the LMS algorithm is not subject to choice, the above results are suggestive 


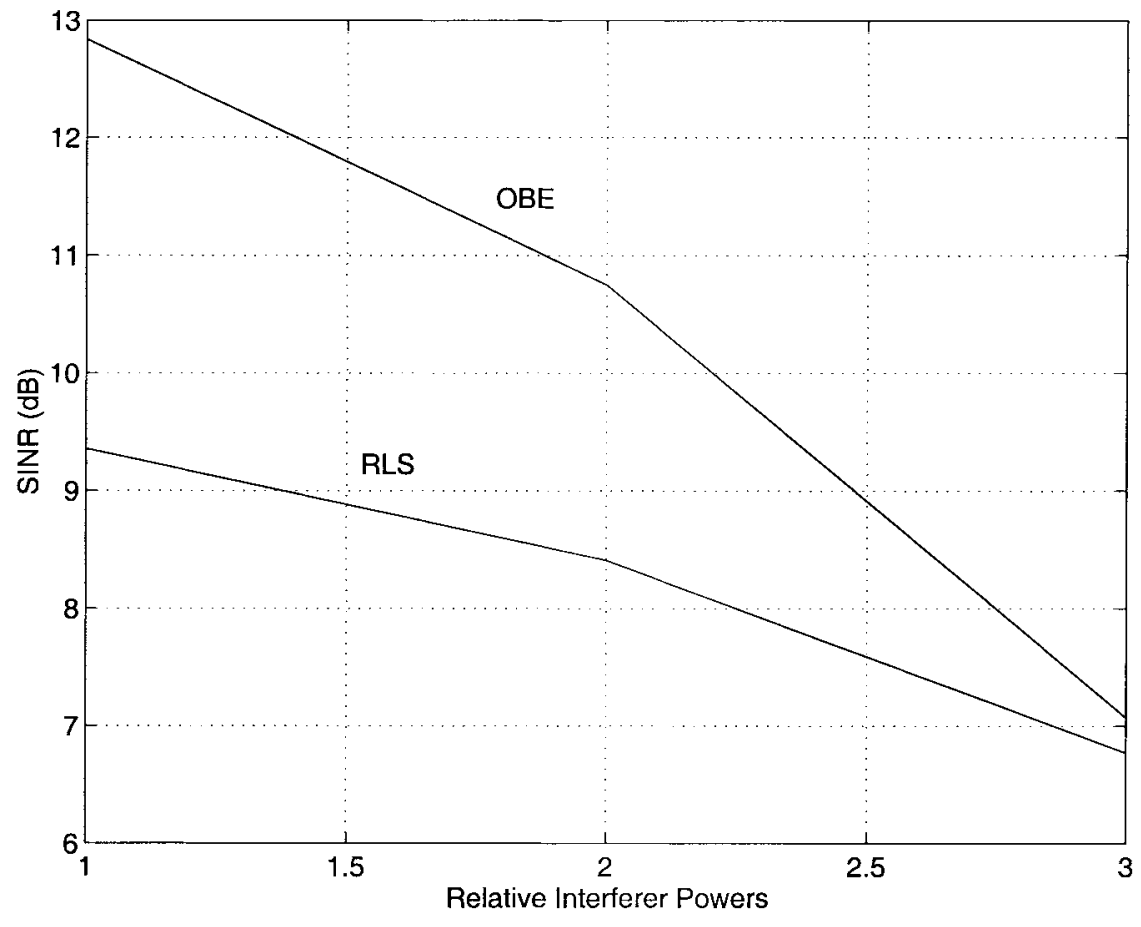

Fig. 9. SINR using OBE and RLS detectors versus relative powers of ten equal power interferers and $N=16$ and $M=2$.

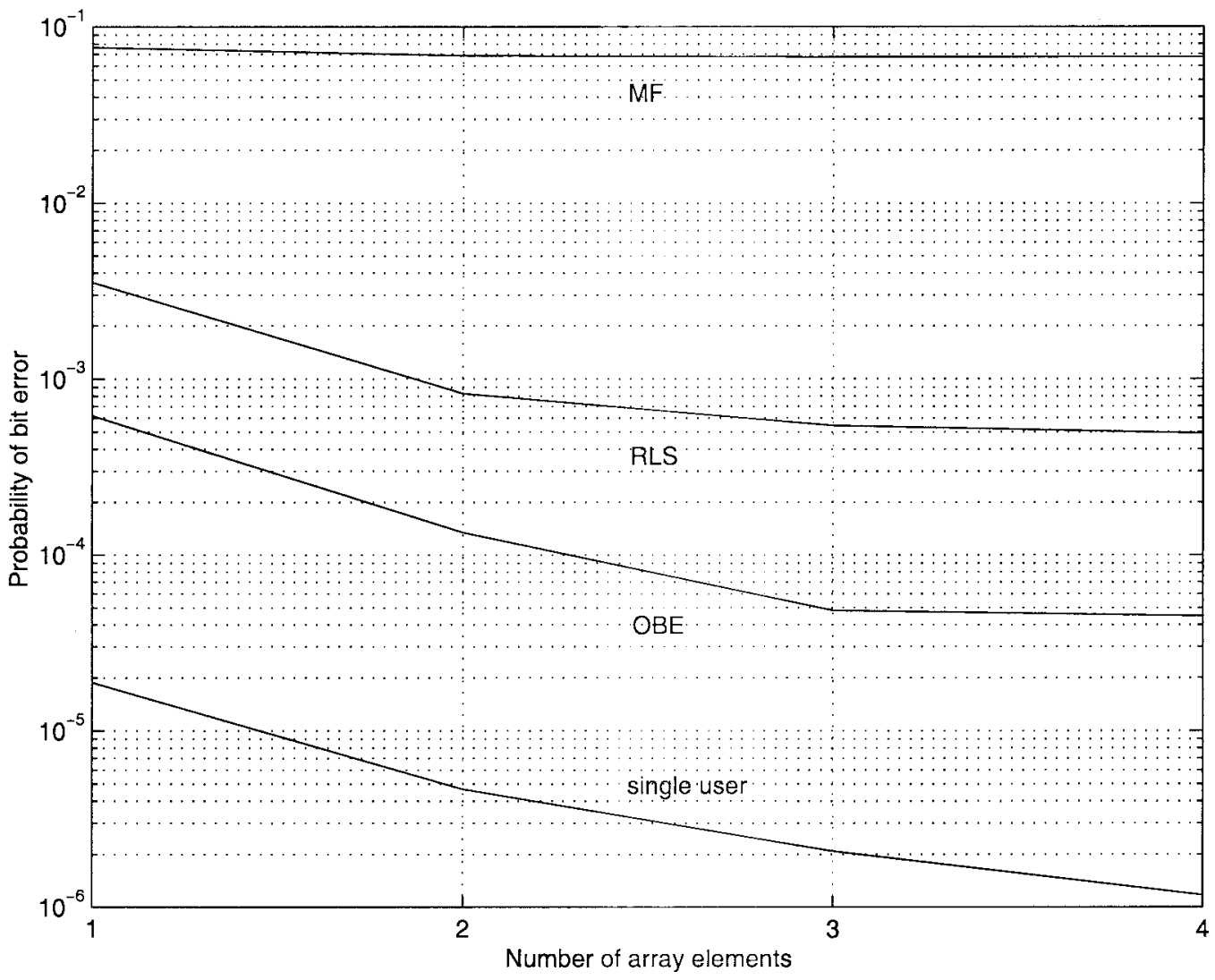

Fig. 10. $P_{b}$ using RAKE receiver versus number of array elements with ten interferers, $L=2$ and $N=32$.

of using a variable step size LMS algorithm which can adapt to time-varying interference conditions. The use of an antenna array at the base station with even two or four elements is seen to be very beneficial for DS-CDMA reception, especially when coupled with multiuser detection. This allows for improved DOA estimates and interference suppression by the beamformer. The benefits of increased SINR due to combined multiuser detection and beamforming can be traded off for more efficient utilization of the uplink spectrum, simplified power control, or combinations thereof. 


\section{CONCLUSIONS}

This paper has presented a new receiver structure for CCI suppression and fading compensation for CDMA signaling over frequency-flat or frequency-selective slowly fading channels. This is accomplished by combining multiuser detection, beamforming, and RAKE reception in a single integrated receiver. Conventional RAKE reception is used to combat multipath fading while CCI suppression is carried out by multiuser detector and beamforming. $(N-1+M)$ adaptive weights are used for the multiuser detector and beamformer, where $N$ is the spread-spectrum processing gain and $M$ is the number of antenna array elements. The blind adaptive multiuser detector is formulated using a constrained energy minimization criterion and adaptation is carried out using a novel OBE algorithm. The OBE multiuser detector provides fast convergence and superior tracking relative to conventional adaptive algorithms such as LMS and RLS. Also, a simple and robust approximate maximum-likelihood DOA estimator is presented for beamforming.

\section{APPENDIX A}

\section{SPATIO-TEMPORAL IMPULSE RESPONSE}

The composite spatio-temporal impulse response of the channel and the $m$ th sensor to the $k$ th-user's signals is given by

$$
h_{k}^{(m)}(t)=\sum_{l=0}^{L-1} a_{k l}^{(m)} \delta\left(t-\tau_{k l} T_{c}\right)
$$

where $a_{k l l}^{(m)}$ is the response of the $m$ th antenna element to the $l$ th multipath component from the $k$ th user. Each multipath component is received with an angle spread of $\triangle$ and is assumed to be distinct (nonoverlapping) from all other paths of the same user. The angle spread arises due to a large number of rays emanating from local scatterers in the vicinity of the transmitting source. Each scatterer manifests itself as a subcomponent which is not resolvable from other subcomponents at the receiver due to the small delays [14], [26]. The different multipath components (from different directions), however, are assumed to be delayed by at least one chip time allowing them to be resolved by RAKE branches. In other words, each RAKE branch sees a distinct flat-fading signal with a certain angle spread. Thus

$$
a_{k l}^{(m)}=\sum_{i} p_{k l i} e^{j\left(q_{k l i}+\phi_{k l i}^{(m)}\right)}
$$

where the summation is taken over all the subcomponents; $\phi_{k l i}^{(m)}$ denotes the angular deviation of the spatial angle of the $i$ th subcomponent of the $l$ th multipath component; and $p_{k l i}$ and $q_{k l i}$ denote the corresponding channel magnitude and phase response, respectively. The spatial angle $\phi_{k l i}^{(m)}$ is given by

$$
\phi_{k l i}^{(m)}=2 \pi(m \rho / v) \sin \left(\theta_{k l}+\delta \theta_{k l i}\right)
$$

where $\theta_{k l}$ denotes the nominal angle-of-arrival of the $k$ thusers' $l$ th multipath component and $\delta \theta_{k l l i}$ is the angular deviation of the $i$ th subcomponent's DOA. For small-angle spread $\triangle$

$$
\phi_{k: l i}^{(m)} \approx \phi_{k l l}^{(m)}+\delta \phi_{k l i}^{(m)}
$$

where

$$
\phi_{k l}^{(m)}=2 \pi(m \rho / v) \sin \left(\theta_{k l}\right)
$$

and

$$
\delta \phi_{k l i}^{(m)}=2 \pi(m \rho / v)\left[\cos \left(\theta_{k l l}\right) \delta \theta_{k l l i}-(1 / 2) \sin \left(\theta_{k l l}\right) \delta \theta_{k l i}^{2}\right] .
$$

Note that for point sources, $\delta \phi_{k l i}^{(m)}=0$. Using (A.2)-(A.5)

$$
a_{k l}^{(m)}=e^{j \phi_{k l}^{(m)}} \sum_{i} p_{k l l i} e^{j q k l i} e^{j \delta \phi_{k l i}^{(m)}}
$$

$\left(\sum_{i} p_{k l i} e^{j q_{k l l}} e^{j \delta \phi_{k l i}^{(m)}}\right)$ is usually modeled as a complex Gaussian random variable since it is the summation of a large number of i.i.d. random variables constituting the channel attenuation for each multipath component.

\section{APPENDIX B \\ DOA-MLE DERIVATION}

Rewriting (4.1) in vector form using $P$ samples for sensor $m$ and $(m+1)$

$$
\begin{aligned}
\boldsymbol{e}_{k l}^{(m)} & =\sqrt{P_{k}} a_{k l}^{(m)} A_{k}+\tilde{\boldsymbol{I}}_{k l}^{(m)} \\
\boldsymbol{e}_{k l}^{(m+1)} & =\sqrt{P_{k}} a_{k l}^{(m+1)} A_{k}+\tilde{\boldsymbol{I}}_{k l}^{(m+1)}
\end{aligned}
$$

where $\boldsymbol{e}_{k l}^{(m)}, \boldsymbol{a}_{k l}^{(m)}, \boldsymbol{A}_{k}$, and $\tilde{\boldsymbol{I}}_{k l}^{(m)}$ denote vectors of length $P$ of the respective temporal samples. Using (A.6)

$$
a_{k l}^{(m+1)}=e^{j \phi_{k l}^{(1)}} a_{k l}^{(m)}+\epsilon_{k l}^{(m+1)}
$$

where $\epsilon_{k: l}^{(m)}$ denotes the residual difference between the channel attenuation at the $m$ th sensor due to angle spreading. To obtain $\epsilon_{k l}^{(m)}$, a series expansion of (A.6) may be carried out for small $\delta \phi_{k l i}^{(m)}$ according to

$$
e^{j \delta \phi_{k l i}^{(m)}} \approx 1+j \delta \phi_{k l i}^{(m)}
$$

Thus yielding

$$
\epsilon_{k l l}^{(m)}=e^{j \phi_{k l}^{(m)}} \sum_{i}\left(j \delta \phi_{k l i}^{(1)}\right) p_{k l i} e^{j q_{k l i}} .
$$

Under isotropic scattering, $\epsilon_{k l .}^{(m)}$ can be regarded as complexvalued zero-mean and Gaussian-distributed. Denoting $\phi_{k l}^{(1)}$ by $\Phi$ for notational simplicity and the conditional probability density function of $\boldsymbol{e}_{k l}^{(m)}$ by $f_{\boldsymbol{e}}, \hat{\Phi}_{\mathrm{ML}}$ is given by

$$
\begin{aligned}
\hat{\Phi}_{\mathrm{ML}} & =\arg \max _{\Phi} f_{e}\left(\boldsymbol{e}_{k l}^{(m+1)}, \boldsymbol{e}_{k l}^{(m)} / \Phi\right) \\
& =\arg \max _{\Phi} f_{e}\left(\boldsymbol{e}_{k l}^{(m+1)} / \boldsymbol{e}_{k l}^{(m)}, \Phi\right)
\end{aligned}
$$

since $e_{k l}^{(m)}$ is independent of $\Phi$. Now, using (B.2) and (B.3)

$$
\boldsymbol{e}_{k l}^{(m+1)}=\boldsymbol{e}_{k l}^{(m)} e^{j \Phi}-\tilde{I}_{k l}^{(m)} e^{j \Phi}+\tilde{\boldsymbol{I}}_{k l}^{(m+1)}+\epsilon_{k l}^{(m+1)} \sqrt{P_{k}} A_{k} .
$$

Conventional assumptions are now made on the postdetection interference vectors to enable use of an ML approach. Namely, they are assumed to be instances of a stationary, ergodic, zeromean complex-valued Gaussian process. Thus the mean of 
$\boldsymbol{e}_{k l l}^{(m+1)}$ is $\left(\boldsymbol{e}_{k l}^{(m)} e^{j \Phi}\right)$ and $f_{\boldsymbol{e}}$ can be factored into a product of partial densities. Thus $\hat{\Phi}_{\mathrm{ML}}$ can be obtained by maximizing the log-likelihood function according to

$\hat{\Phi}_{\mathrm{ML}}=\arg \max _{\Phi}\left[\left(\boldsymbol{e}_{k l}^{(m+1)}-\boldsymbol{e}_{k l}^{(m)} e^{j \Phi}\right)^{H}\left(\boldsymbol{e}_{k l}^{(m+1)}-\boldsymbol{e}_{k l}^{(m)} e^{j \Phi}\right)\right]$.

Differentiating the right-hand side of (B.8) with respect to $\Phi$ and setting to zero yields

$$
\left[\boldsymbol{e}_{k l}^{(m)}\right]^{H} \boldsymbol{e}_{k l l}^{(m+1)} e^{-j \Phi}=\left[\boldsymbol{e}_{k l}^{(m+1)}\right]^{H} \boldsymbol{e}_{k l}^{(m)} e^{j \Phi} .
$$

Noting that the right-hand side of (B.9) is merely the complex conjugate of the left-hand side, and setting the imaginary part to zero yields the desired result in (4.2).

\section{REFERENCES}

[1] K. M. Ahmed and R. J. Evans, "An adaptive array processor with robustness and broad-band capabilities," IEEE Trans. Antennas Propagat., vol. 32, pp. 944-950, Sept. 1984

[2] S. Anderson, M. Millnert, M. Viberg, and B. Wahlberg, "An adaptive array for mobile communication systems," IEEE Trans. Veh. Technol., vol. 40, pp. 230-236, Feb. 1991.

[3] T. A. Brown, "The use of antenna arrays in the detection of code division multiple access signals," Ph.D. dissertation, Univ. Minnesota, Minneapolis, 1995.

[4] P. L. Combettes, "The foundations of set-theoretic estimation," Proc. IEEE, vol. 81, pp. 182-208, Feb. 1993.

[5] S. Dasgupta and Y. F. Huang, "Asymptotically convergent modified recursive least squares with data dependent updating and forgetting factor for systems with bounded noise," IEEE Trans. Inform. Theory, vol. IT-33, pp. 383-392, May 1987.

[6] J. R. Deller and S. F. Odeh, "Adaptive set-membership identification in $\mathcal{O}(m)$ time for linear-in-parameter models," IEEE Trans. Signal Processing, vol. 41, pp. 1906-1924, May 1993.

[7] J. R. Deller, M. Nayeri, and S. F. Odeh, "Least square identification with error bounds for real-time signal processing and control," Proc. IEEE, vol. 81, pp. 813-849, June 1993.

[8] E. Fogel and Y. F. Huang, "On the value of information in system identification-bounded noise case," Automatica, vol. 18, no. 2, pp. 229-238, Mar. 1982.

[9] V. Ghazi-Moghadam, M. Kaveh, and L. Nelson, "A multi-element blind adaptive multiuser detector," in Proc. IEEE Signal Processing Worksh. Statistical Signal and Array Processing, Corfu, Greece, June 1996.

[10] S. Gollamudi, S. Kapoor, S. Nagaraj, and Y. F. Huang, "Set-membership adaptive equalization and an updator-shared implementation for multiple channel communication systems," IEEE Trans. Signal Processing, vol. 46, pp. 2372-2385, Sept. 1998

[11] S. Gollamudi, S. Nagaraj, S. Kapoor, and Y. F. Huang, "Set-membership filtering and a set-membership normalized LMS algorithm with adaptive step size," IEEE Signal Processing Lett., pp. 111-114, May 1998.

[12] S. Haykin, Adaptive Filter Theory. Englewood Cliffs, NJ: PrenticeHall, 1996.

[13] M. L. Honig, U. Madhow, and S. Verdú, "Blind adaptive multiuser detection," IEEE Trans. Inform. Theory, vol. 41, pp. 944-960, July 1995.

[14] W. C. Jakes, Microwave Mobile Communications. New York: IEEE Press, 1994.

[15] S. Kapoor, S. Gollamudi, S. Nagaraj, and Y. F. Huang, "Tracking of time-varying parameters using optimal bounding ellipsoid algorithms," in Proc. 34th Annu. Allerton Conf. Comm.unication Control and Components, Monticello, IL, Oct. 1996.

[16] S. Kapoor and Y. F. Huang, "Blind multiuser detection and interference cancellation in DS-CDMA mobile radio systems," in Proc. IEEE Worksh. Signal Procrocessing Advances in Wireless Communication (SPAWC'97), Paris, France, Apr. 1997.

[17] R. Kohno et al., "Combination of an adaptive array antenna and a canceler of interference of direct-sequence spread-spectrum multipleaccess system," IEEE J. Select. Areas Commun., vol. 8, pp. 675-681, May 1990.

[18] U. Madhow, "Signal processing for interference suppression in DSCDMA systems," in Proc. ICASSP'96, Atlanta, GA, May 1996.

[19] R. L. Moses, T. Soderstrom, and J. Sorelius, "Effects of multipath induced angular spread on DOA estimators in array signal processing," in Proc. IEEE/IEE Worksh. Signal Processing Methods in Multipath Environments, Glasgow, Scotland, Apr. 1995.

[20] S. Nagaraj, S. Gollamudi, S. Kapoor, Y. Huang, and J. Deller "Multiuser detection based on a deterministic error specification: Theory and lowcomplexity adaptive solutions," in Proc. 31st Asilomar Conf. Signals, Systems and Computers, Pacific Grove, CA, Nov. 1997.

[21] A. F. Naguib and A. Paulraj, "Performance of CDMA Cellular Networks with Base Station Antenna Arrays," in Proc. Int. Zurich Seminar Digital Communication, Zurich, Switzerland, Mar. 1994, pp. 87-100.

[22] _ "Performance of wireless CDMA with $M$-ary orthogonal modulation and cell site antenna arrays," IEEE J. Select. Areas. Commun., vol. 14, pp. 1770-1783, Dec. 1996.

[23] K. Pahlavan and A. Levesque, Wireless Information Networks. New York: Wiley, 1995.

[24] A. K. Rao and Y. F. Huang, "Tracking characteristics of an OBE parameter estimation algorithm," IEEE Trans. Signal Processing, vol. 41, pp. 1140-1148, Mar. 1993.

[25] R. Roy and T. Kailath, "ESPRIT - Estimation of signal parameters via rotational invariance techniques," IEEE Trans. Acoust., Speech Signal Processing, vol. 37, pp. 984-995, July 1989.

[26] J. Salz and J. H. Winters, "Effect of fading correlation on adaptive arrays in digital mobile radio," IEEE Trans. Veh. Technol., vol. 43, pp. 1049-1057, Nov. 1994.

[27] J. B. Schodorf and D. B. Williams, "A constrained adaptive diversity combiner for interference suppression in CDMA systems," in Proc. ICASSP'96, Atlanta, GA, May 1996.

[28] B. D. Van Veen and K. M. Buckley, "Beamforming: A versatile approach to spatial filtering," IEEE ASSP Mag., vol. 5, pp. 4-24, Apr. 1988.

[29] S. Verdú, "Multiuser detection," in Advances in Statistical Signal Processing, vol. 2. Greenwich, CT: JAI Press, 1993, pp. 369-409.

[30] X. Wang and H. V. Poor, "Blind equalization and multiuser detection in dispersive CDMA channels," IEEE Trans. Commun, vol. 46, pp. 91-103, Jan. 1998.

[31] I. Ziskind and M. Wax, "Maximum likelihood localization of multiple sources by alternating projection," IEEE Trans. Acoust., Speech Signal Processing, vol. 36, pp. 1553-1560, Oct. 1988.

[32] Z. Zvonar, "Combined multiuser detection and diversity reception for wireless CDMA systems," IEEE Trans. Veh. Technol., vol. 45, pp. 205-211, Feb. 1996

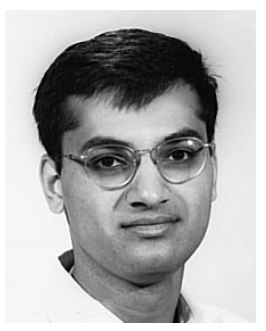

Samir Kapoor (M'98) received the B.Tech., M.S., and $\mathrm{Ph} . \mathrm{D}$. degrees in electrical engineering from the Indian Institute of Technology, Bombay, in 1992, Washington State University, Seattle, in 1994, and University of Notre Dame, Notre Dame, IN, in 1998, respectively.

From 1994 to 1998, he was with Tellabs Research Center Mishawaka, IN. He is currently with NEC USA, Inc., Princeton, NJ. His research interests include array processing, adaptive signal processing, and digital communications.

Dr. Kapoor is a member of Tau Beta Pi and Eta Kappa Nu. He currently serves as an Associate Editor for the IEEE Transactions on Multimedia.

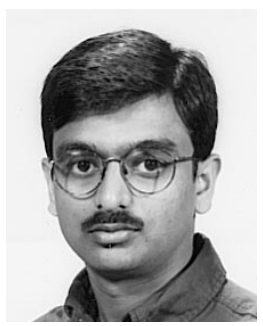

Sridhar Gollamudi (S'95-M'99) received the B.Tech. degree in electrical engineering from the Indian Institute of Technology, Bombay, in May, 1994, and the M.S.E.E. degree from University of Notre Dame, Notre Dame, IN, in January, 1996. He was awarded the Center for Applied Mathematics Fellowship at the University of Notre Dame for the academic year 1996-1997. He is currently working towards the Ph.D. degree in the Department of Electrical Engineering, University of Notre Dame.

From May to December 1997, he was an intern at Lucent Technologies-Bell Laboratories, Whippany, NJ, where he worked on interference suppression for wireless CDMA systems. His research interests are in the areas of statistical and adaptive signal processing, communication theory, multiuser communications, and antenna array processing.

Mr. Gollamudi is a member of Eta Kappa Nu. 


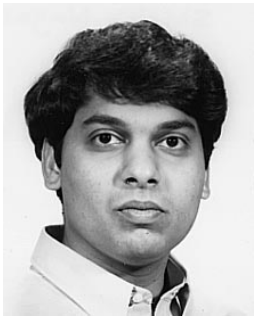

Shirish Nagaraj (M'95-S'95) received the B.Tech. degree from the Indian Institute of Technology, Bombay, in May 1995 and the M.S. degree from University of Notre Dame, Notre Dame, IN, in May, 1997, both in electrical engineering. He is currently working towards the Ph.D. degree at the University of Notre Dame.

He was an intern with Lucent Technologies-Bell Laboratories, Whippany, NJ, from June to December 1998. His research interests include adaptive signal processing, statistical estimation and detection theory, set-membership filtering, and multiuser communications.

Mr. Nagaraj received the Michael J. Birck fellowship during 1996-1997 and a graduate fellowship from the Center for Applied Mathematics, University of Notre Dame, during 1997-1998.

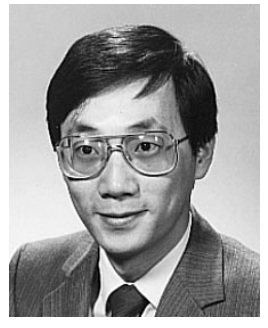

Yih-Fang Huang (S'80-M'82-SM'94-F'95) received the B.S. degree in electrical engineering from National Taiwan University, Tainan, in June, 1976, the M.S.E.E. degree from University of Notre Dame, Notre Dame, IN, in January 1980, and the Ph.D. degree in electrical engineering from Princeton University, Princeton, NJ, in October, 1982.

Since August 1982, he has been on the Faculty at University of Notre Dame where he is currently Professor and Chair of Electrical Engineering. In Spring 1993, he received the Toshiba Fellowship and was Toshiba Visiting Professor in the Department of Electrical Engineering at Waseda University, Tokyo, Japan. His research interests are in the general areas of statistical communications and signal processing. $\mathrm{He}$ also worked on image source coding and more recently joint source-channel coding.

Dr. Huang has served as Associate Editor for the IEEE TRANSACTIONS ON CiRCUITS AND Systems (1989-1991) and for EXPRESS LeTtERS for the same journal during 1992-1993. During 1995-1996, he was Chairman for the Digital Signal Processing Technical Committee of the IEEE Circuits and Systems Society. He was a Cochairman for Workshops/Short Courses for the 1997 International Symposium on Circuits and Systems. He served as Regional Editor of America for the Journal of Circuits, Systems, and Computers. He also served as Vice President-Publications for the IEEE Circuits and Systems Society (1997-1998). 